\title{
Incentives, ability and disutility of effort
}

\section{Silvia Martinez-Gorricho ${ }^{1}$ (D) $\cdot$ Miguel Sanchez Villalba $^{2}$ (C)}

Received: 6 February 2020 / Accepted: 7 May 2021 / Published online: 12 June 2021

(c) The Author(s) 2021

\begin{abstract}
We generalize the disutility of effort function in the linear-Constant Absolute Risk Aversion (CARA) pure moral hazard model. We assume that agents are heterogeneous in ability. Each agent's ability is observable and treated as a parameter that indexes the disutility of effort associated with the task performed. In opposition to the literature (the "traditional" scenario), we find a new, "novel" scenario, in which a high-ability agent may be offered a weaker incentive contract than a low-ability one, but works harder. We characterize the conditions for the existence of these two scenarios: formally, the "traditional" ("novel") scenario occurs if and only if the marginal rate of substitution of the marginal disutility of effort function is increasing (decreasing) in effort when evaluated at the second-best effort. If, further, this condition holds for all parameter values and matching is endogenous, less (more) talented agents work for principals with riskier projects in equilibrium. This implies that the indirect and total effects of risk on incentives are negative under monotone assortative matching.
\end{abstract}

Keywords Incentives · Ability · Disutility of effort · Endogenous matching $\cdot$ Moral hazard

JEL Classification C78 · D03 - D82 · D86

Silvia Martinez-Gorricho

smartinez@ucsc.cl

Miguel Sanchez Villalba

miguelsanchez@udec.cl

1 Department of Economics, Universidad Católica de la Santísima Concepción, Alonso de Ribera 2850, Concepción, Chile

2 Department of Economics, Universidad de Concepción, Victoria 471, Concepción, Chile 


\section{Introduction}

When agents are heterogeneous in ability, the optimal contract offered by the principal will typically depend on the ability/talent of the agent she faces. ${ }^{1}$ But will incentives be stronger for low- or for high-ability agents? The aim of our paper is to offer insights regarding the determinants of compensation schemes, the design of incentives and the sorting of employees and employers - issues that have long been central to the agency literature. So far, this literature has yielded what we label as the "traditional" scenario, namely, that more talented agents are offered contracts with stronger incentives ( $\mathrm{Li}$ and Ueda 2009; Bandiera et al. 2015; Kandilov and Vukina 2016; Wulf 2007) and exert more effort than less talented ones (Li and Ueda 2009; Bandiera et al. 2015; Chade and Swinkels 2020). ${ }^{2}$ We show that a version of the Holmstrom and Milgrom (1987)'s linear-CARA moral hazard model with quadratic costs in which agents are heterogeneous in terms of their cost of effort (henceforth, the "canonical model") also predicts that the higher the ability of the agent is, the higher is the efficiency loss caused by the associated inefficient risk sharing, despite a lower effort-distortion rate. We prove that the previous predictions rely on the assumptions of a multiplicatively separable and homogeneous (in effort) disutility of effort function. However, if a more general cost function is considered, the model allows for a second, "novel" scenario-overlooked by the literature — in which more talented agents are offered weaker incentive schemes. In line with previous research (Li and Ueda 2009; Chade and Swinkels 2020), we assume that agents are heterogeneous in their ability level, which is public information and parameterizes the disutility or cost of effort (COE) associated with the activity performed at the workplace, and that the more talented the agent is, the lower the associated COE is. ${ }^{3}$ We depart from the "canonical model" by generalizing the $\mathrm{COE}$ function and we find that the strength of incentives, the efficiency loss and the effort distortion need not to individually increase with ability or to move in unison.

Our main contribution to the agency literature is that we find a necessary and sufficient condition on the properties of the original feature in our model, namely the generalized COE function, that determines whether the "traditional" or "novel" scenario will occur (formally, it identifies the sign of the effect of ability on the strength of incentives under pure moral hazard) and that, further, determines the sorting pattern

\footnotetext{
1 Throughout the paper, and without loss of generality, we will assume that the principal is female and the agent is male.

2 Specifically, please refer to the sensitivity analysis example developed by Holmstrom and Milgrom (1987) on page 323. A quadratic-in-effort cost structure is also assumed in Li and Ueda (2009), Bandiera et al. (2015) and Kandilov and Vukina (2016).

3 In contrast, the disutility of effort is assumed only dependent on effort in Edmans and Gabaix (2011), Bandiera et al. (2015) and Kandilov and Vukina (2016). In Bandiera et al. (2015) and Kandilov and Vukina (2016), a manager's higher ability is assumed to increase his productivity, instead of decreasing his COE, and the COE is assumed to be quadratic in effort. In Edmans and Gabaix (2011), managers are assumed to have CRRA utility functions instead, and hence, utility is multiplicative in wage and effort. As wealthier managers are more willing to forgo incentive pay to enjoy leisure, exerting any given level of effort is more costly to the manager the wealthier (i.e., more talented) he is. Furthermore, in Edmans and Gabaix (2011), firms (principals) are assumed to differ in size rather than risk, whereas they differ in ownership in Bandiera et al. (2015).
} 
that ensues in equilibrium under endogenous matching. Specifically, we prove that the "traditional" ("novel") scenario occurs if and only if the absolute value of the marginal COE's marginal rate of substitution between ability and effort is increasing (decreasing) in effort when evaluated at the second-best solution. We refer to this as the increasing (diminishing) marginal rate of substitution condition (henceforth the IMRSC (DMRSC)).

In our framework, by the complementarity of effort and ability in the principal's maximization problem, principals prefer to hire high-ability agents to low-ability ones if agents' reservation utilities are not very sensitive to ability. In addition, principals induce more talented agents to exert a strictly higher level of effort than less talented ones. In the second-best contract, the power is set equal to the marginal $\mathrm{COE}$ at the second-best effort. As it is standard in the literature (Li and Ueda 2009; Chade and Swinkels 2020), we assume that the marginal cost of exerting any fixed level of effort is strictly higher for less talented agents than more talented ones. Hence, the direct effect of ability on incentives is to reduce the power since providing incentives to more talented agents is less expensive. However, since the second-best effort increases with ability and the marginal COE is increasing in effort, the indirect effect of ability on incentives leads to an increase in the power of the scheme: the higher effort induced to the more talented agent makes his provision of incentives more expensive.

Since the marginal rate of substitution (MRS) of the marginal COE function reflects the relative sensitivity of said function to ability vis-a-vis effort, large (small) values indicate that agents are (not) very responsive to changes in ability. Furthermore, in equilibrium, the MRS denotes the direct effect of ability on effort, for a fixed power of the incentive scheme. Under the IMRSC, the second-best effort is sufficiently responsive to ability so that the indirect effect dominates the direct effect, leading to a strictly higher power for more talented agents. Since a multiplicatively separable marginal COE function that is log-concave in effort everywhere implies the IMRSC, the prediction of an increasing in ability power by the "canonical model" follows immediately. On the contrary, since the second-best effort is not sufficiently responsive to ability under the DMRSC, the direct effect overwhelms the indirect effect and the power is decreasing in ability. Furthermore, it is not necessary for the IMRSC/DMRSC to hold everywhere, and we illustrate this with an example (Example 2) in which the power of the incentive scheme is non-monotonic in ability: the optimal sharing rule is an inverted U-shaped function of ability since the marginal COE satisfies the IMRSC for relatively low levels of effort (which are exerted by less talented agents in equilibrium), whereas it satisfies the DMRSC for relatively high levels of effort (which are exerted by more talented agents in equilibrium).

Our results suggest that the IMRSC/DMRSC is crucial to determine the equilibrium compensation package and, as we will see next, the sorting in the labor market. Controlling for risk, Wulf (2007) finds that pay-performance sensitivities (i.e., the power of incentive scheme) vary by authority across senior division managers in the same job classification in large firms. Interpreting a manager with broader authority as a high-ability division manager, pay is found almost four times more sensitive to performance (firm sales growth) for high-ability managers than for low-ability ones 
(i.e., officer versus non-officer). ${ }^{4}$ This evidence is consistent with the performance of managerial activities associated with a marginal COE that satisfies the IMRSC. However, data sets usually include a panel of firms that spans several industries. ${ }^{5}$ Consider the following thought experiment in which two firms with manager positions in the same job classifications, similar performance and variance of returns, operate in different sectors. Specifically, suppose that the marginal COE associated with the typical managerial activity/task performed in firm 1 (firm 2) satisfies the IMRSC (DMRSC). If we run a regression of pay on performance, controlling only for the variance of the firm and the managers' abilities, it will incorrectly find no significant effect of ability on performance: since the more able managers are offered a higher powered incentive contract than the less able managers in firm 1 but the reverse holds true in firm 2, the estimated coefficient of ability will be biased toward zero, as the model ignores the role of the shape of the marginal COE. In order to avoid such bias in the estimation, and in addition to controlling for fixed effects, the inclusion of an interaction term between industry/firm indicators, performance and ability in the empirical contract choice equation would be highly recommended, as it would help to control for the effect of the shape of the marginal COE structure on incentives. Consider now a second thought experiment with firms operating in similar sectors. Suppose that the marginal COE associated with the typical task performed by less talented managers satisfies the IMRSC but the marginal COE associated with the typical task performed by more talented managers satisfies the DRMSC (or vice-versa). Our model predicts an optimal sharing rule that is non-monotonic in ability: the strongest (weakest) incentives are offered to a manager with an intermediate level of ability. As the data contain discrete measures of managers' ability which are usually highly correlated with a job/activity indicator of the management position (e.g., officers versus non-officers managers), it may be possible that no performance sensitivity to ability is again spuriously found in the estimation. In this case, the use of instruments for ability different from job indicators and the estimation of a polynomial regression of higher degree in ability is highly encouraged. In sum, we highlight that the nature of the agent's task-in essence, the shape of the marginal COE structure associated with the activity performed-is crucial when assessing the relationship between risk, ability and incentives in empirical work.

In our model we assume-as is standard in the literature - that the only source of heterogeneity on the principal's side is risk. Having that in mind, consider now an industrial sector in which the typical (managerial) activities are characterized by a marginal COE function that satisfies the IMRSC (DMRSC) for all parameter values. Then, we show that positive (negative) assortative matching on ability ensues in equilibrium for all type distributions: principals with riskier projects are pairwise-matched with less (more) talented agents and offer them weaker incentives. In sum, the negative indirect effect of risk on incentives reinforces the direct effect in both scenarios characterized by monotone matching. However, the direct and indirect effects of risk on

\footnotetext{
4 On average, $23 \%$ of division managers are corporate officers. A similar conclusion is obtained by Aggarwal and Samwick (1999) who find that at the median variance, estimated pay-performance sensitivities of top executives who are not identified as CEOs are about one-fifth the size of those for CEOs.

5 For instance, the majority of the firms in Wulf (2007)'s data set operate in the food, paper, chemical, machinery, electrical, transportation equipment, instrumentation, communications and utilities industries.
} 
the optimal effort only reinforce each other under positive assortative matching. Our model predicts that in an equilibrium characterized by negative assortative matching, more talented agents exert higher (lower) levels of effort than less talented ones if and only if the matching function is sufficiently steep (flat).

To the best of our knowledge, our findings are novel in the literature, reviewed recently by Macho-Stadler and Pérez-Castrillo (2018) and Macho-Stadler and PérezCastrillo (2020). ${ }^{6}$ Positive sorting in equilibrium is predicted by Li and Ueda (2009) and Chade and Swinkels (2020). Li and Ueda (2009) apply the "canonical model" to an endogenous matching framework. ${ }^{7}$ More broadly, we show that the sorting pattern that ensues in equilibrium depends on the properties of the marginal $\mathrm{COE}$ associated with the performed activities, and hence, that negative sorting is plausible for some COE structures (i.e., some tasks). Chade and Swinkels (2020) generalize the COE function in a framework with hyperbolic absolute risk aversion (i.e., imperfect transferable) utility functions. But while their risk preferences are more general than ours, the validity of their results requires a sufficiently attractive outside option for the agent. Furthermore, the authors center their research on performing comparative statics of the second-best effort with respect to some variables of interest such as the agent's ability or his initial wealth. In contrast, we focus on the sign of the relationship between incentives, ability and risk. The extra information structure imposed by the linear contract and the CARA risk-preferences assumptions which imply a perfectly transferable utility, allows us to make more predictions in a competitive market setting.

Finally, Serfes (2005) performs a similar theoretical analysis applying the "canonical model" but assuming that agents differ in their degree of risk aversion, instead of ability. ${ }^{8} \mathrm{He}$ finds that a positive relationship between incentives and risk is plausible only under negative assortative matching since the partial effect of both variables (risk and risk aversion) on the incentive power is negative via the optimal induced effort. Although a negative sorting pattern is a possible prediction of our model, we find that the total effect of risk on power is unambiguously negative under monotone assortative matching if agents are heterogeneous only in ability. This result is due to the positive correlation between the sign of the total effect of ability on the incentive power and the sorting pattern that emerges in equilibrium. We show that the indirect effect of risk on incentives is locally negative for almost all parameter values even when the equilibrium assignment is not globally assortative. Thus, a globally non-monotonic relationship between incentives and risk could be obtained only if the matching function is discontinuous. The negative relationship between risk and incentives found in our paper under endogenous matching is consistent with some of the evidence found in the literature (Wulf 2007; Hilt 2006; Aggarwal and Samwick 1999). Ackerberg

\footnotetext{
6 For an overview of the personnel economics literature refer to Lazear and Oyer (2012).

7 The authors also consider the case of multidimensional agents who are heterogeneous not only in their ability but also in their degree of risk aversion. They specifically assume that more able agents are at least as risk tolerant as less able agents. Refer also to Bandiera et al. (2015) for a framework in which agents (e.g., managers) are uniformly distributed on a two-dimensional space of ability and risk-aversion.

8 Chen et al. (2018) discuss endogenous matching between risk-averse principals and risk-averse agents with endogenous risk as agents can make efforts not only to control the mean but also the idiosyncratic risk of the output. They find that the presence of moral hazard leads to positive assortative matching in equilibrium.
} 
and Botticini (2002) offered endogenous matching as an explanation for the puzzling mixed empirical evidence on the relationship of risk and incentives. They point out that empirical studies that ignore endogenous matching are likely to generate potentially significant biases (in sign and magnitude) in the estimated coefficients of the compensation-related independent variables. ${ }^{9}$

Our paper is organized as follows. The framework is described in Sect. 2. Section 3 computes the optimal policies associated with each informational setting. Section 4 presents the main results of the paper in an isolated principal-agent relationship. Section 5 analyzes sorting in a general equilibrium setting and studies the effect of risk on incentives and effort under monotone assortative endogenous matching. Section 6 briefly concludes the paper. All relevant proofs are contained in "Appendix."

\section{The baseline model}

A male risk-averse agent, characterized by his ability level, meets a female risk-neutral principal, characterized by the risk (variance) of her project $\sigma^{2}$. The principal wishes to hire the agent to perform a task (e.g., managerial activity) in exchange for a wage $w$. The output of the relationship, denoted by $x \in X$, is informative about the hidden effort exerted by the agent, $a \in\left[a_{L}, a_{H}\right]:=A$ with $a_{L} \geq 0 .{ }^{10}$ Specifically, $x=a+\varepsilon$ where $\varepsilon \sim N\left(0, \sigma^{2}\right)$ and $\sigma^{2}<\infty$. We modify the canonical CARA-principal-agent model by generalizing the COE function associated with the task executed $v(k, a)$, measured in monetary units, where parameter $k \in\left[k_{L}, k_{H}\right]:=K$, with $k_{L}>0$, is an inverse measure of the agent's ability type: the most (least) talented agent is indexed by $k_{L}\left(k_{H}\right) .{ }^{11}$ We assume that the function $v$ and the values $\left(k, \sigma^{2}\right)$ are public information to abstract from hidden information issues. ${ }^{12}$

Assumption 1 Function $v$ is thrice continuously differentiable, with $v\left(k, a_{L}\right)=0$ for all $k \in K, v_{a}>0, v_{k}>0, v_{a k}>0, v_{a a}>0$ and $v_{a a k} \geq 0$ for all $(k, a) \in K \times A$ such that $a>a_{L}$. Furthermore, $a_{H} \geq v\left(k_{H}, a_{H}\right), v_{a}\left(k_{H}, a_{L}\right)<1<v_{a}\left(k_{L}, a_{H}\right)$ and $v_{a a}^{2}+v_{a} v_{a a a}>0$ for all $(k, a) \in K \times\left(a_{L}, a_{H}\right]$.

Under Assumption 1, the COE function is increasing and convex in effort but less able agents experiment a higher total and marginal disutility of effort than their more able counterparts. Similarly, their disutility is more convex in effort. The inequality $v_{a}\left(k_{H}, a_{L}\right)<1<v_{a}\left(k_{L}, a_{H}\right)$ ensures that the optimal effort is interior in the firstbest scenario. Assumption $a_{H} \geq v\left(k_{H}, a_{H}\right)$ guarantees that hiring (an agent with any ability level) is efficient when effort is verifiable since it generates a strictly positive

\footnotetext{
9 The endogenous matching generates correlation between the observable characteristic (risk) of the principals and proxy errors of the agents' imperfectly observable characteristic. Prendergast (2002) provides some evidence of a positive relationship between risk and incentives across fields.

10 More generally, the results also hold if the action set is continuous but not compact, as long as Assumption 1 is satisfied (taking limits).

11 As in Li and Ueda (2009), we use an inverse measure of ability for comparison purposes.

12 Henceforth, for any given function $f$, we denote its partial derivative with respect to variable $x$ by $f_{x}$ and its elasticity with respect to variable $x$ by $\epsilon_{f, x}: \epsilon_{f, x}:=\left(x f_{x}\right) / f$. If the function is of a single variable, its derivative is simply denoted by $f^{\prime}$.
} 
expected surplus. The last inequality in Assumption 1 guarantees that the principal's profit maximization problem is well-behaved. Note that the quadratic COE function specified in the canonical CARA model, $v(k, a)=(1 / 2) k a^{2}$ with $a_{L}=0$, satisfies Assumption 1 if and only if $a_{H} \in\left(1 / k_{L}, 2 / k_{H}\right]$. Henceforth, we will refer to $v_{a}(k, a)$ as the marginal COE function.

The agent has CARA risk preferences, with $u(w, k, a)=-\exp \{-r(w-v(k, a))\}$, where $r>0$ denotes his Arrow-Pratt coefficient (of absolute risk aversion). He has a reservation certainty-equivalent outside the relationship with the principal given by $\underline{\mathrm{CE}}(k)$ which may be type-dependent (e.g., $\left.\mathrm{CE}^{\prime}(k) \leq 0\right)$. The principal's objective is to maximize the expected net profit given by $a-E(w)$. We restrict attention to linear compensation schemes of the form $w(x)=w_{0}+s x$, where $s \in \mathbb{R}$ is the power (e.g., pay-performance sensitivity) and $w_{0} \in \mathbb{R}$ is the fixed part of the incentive scheme. ${ }^{13}$

The timing of the contracting problem is as follows: (1) the principal offers a linear wage scheme $\left(w_{0}, s\right)$ to the agent; (2) the agent decides whether to accept the offered contract and if so, executes a hidden action; (3) if the contract has been accepted, output is publicly observed and the agent is paid accordingly. Thus, the principal assumes all the bargaining power and the agent is willing to accept the contract $\left(w_{0}, s\right)$ as long as it guarantees him his reservation utility. The agent's certainty-equivalent income of accepting the contract $\left(w_{0}, s\right)$ is given by $\operatorname{CE}\left(k, a, w_{0}, s\right):=w_{0}+s a-$ $v(k, a)-(1 / 2) r \sigma^{2} s^{2}$ and the principal's expected profits are given by $\Pi\left(a, w_{0}, s\right)=$ $a(1-s)-w_{0}$.

\section{Optimal policy in an isolated partnership}

The following results are sketched briefly as they are standard.

\subsection{First-best}

When effort is verifiable, the first-best effort $a^{\mathrm{FB}} \in\left(a_{L}, a_{H}\right)$ is interior and it satisfies

$$
v_{a}\left(k, a^{\mathrm{FB}}\right)=1
$$

Due to the agent's risk-aversion, the first-best policy yields full insurance $\left(s^{\mathrm{FB}}=0\right)$ with a fixed payment given by $w_{0}^{\mathrm{FB}}=v\left(k, a^{\mathrm{FB}}\right)+\underline{\mathrm{CE}}(k)$ if $a=a^{\mathrm{FB}}$ and zero otherwise, leading to a Pareto efficient outcome. The principal's expected profits are $\Pi^{\mathrm{FB}}(k)=a^{\mathrm{FB}}-v\left(k, a^{\mathrm{FB}}\right)-\underline{\mathrm{CE}}(k)$.

\subsection{Second-best}

By the First-Order Approach, the Incentive Compatibility Constraint (ICC) can be replaced by the interior solution to the agent's maximization problem, $s=v_{a}(k, a)$,

\footnotetext{
13 Holmstrom and Milgrom (1987) show that the optimal contract is linear in output in a dynamic version of the model in which the agent controls the drift vector with a Brownian motion process and observes his accumulated performance before acting.
} 
leading to a strictly positive power. ${ }^{14}$ Therefore, the provision of incentives prevents the provision of full insurance as is well-known in the literature. The induced interior second-best effort level $a^{\mathrm{SB}}$ satisfies:

$$
v_{a}\left(k, a^{\mathrm{SB}}\right)\left[1+r \sigma^{2} v_{a a}\left(k, a^{\mathrm{SB}}\right)\right]=1
$$

We assume that the value $r \sigma^{2}$ is such that the solution to Equation (2) is interior (in other words, it is never satisfied at $a_{L}$ ). If the agent is risk averse, the moral hazard problem leads to under provision of effort in equilibrium: $v_{a a}(k, a)>0$ for any given pair $(k, a) \in K \times A$ and Equations (1) and (2) imply $v_{a}\left(k, a^{\mathrm{SB}}\right)<1=v_{a}\left(k, a^{\mathrm{FB}}\right)$, and hence, $a_{L} \leq a^{\mathrm{SB}}<a^{\mathrm{FB}}$. Thus, given the asymmetry of information and the noisiness of the outcome, the provision of incentives distorts the efficient allocation of risk in the relationship and the efficient allocation of effort by the agent. Indeed,

$$
\begin{aligned}
s^{\mathrm{SB}} & =v_{a}\left(k, a^{\mathrm{SB}}\right) \\
w_{0}^{\mathrm{SB}} & =v\left(k, a^{\mathrm{SB}}\right)-v_{a}\left(k, a^{\mathrm{SB}}\right) a^{\mathrm{SB}}+\frac{1}{2} r \sigma^{2} v_{a}^{2}\left(k, a^{\mathrm{SB}}\right)+\underline{\mathrm{CE}}(k) \\
\Pi^{\mathrm{SB}}\left(k, r, \sigma^{2}\right) & =a^{\mathrm{SB}}-v\left(k, a^{\mathrm{SB}}\right)-\frac{1}{2} r \sigma^{2} v_{a}^{2}\left(k, a^{\mathrm{SB}}\right)-\underline{\mathrm{CE}}(k) \\
S^{\mathrm{SB}}\left(k, r, \sigma^{2}\right) & :=\Pi^{\mathrm{SB}}\left(k, r, \sigma^{2}\right)+\mathrm{CE}^{\mathrm{SB}}\left(k, r, \sigma^{2}\right) \\
& =a^{\mathrm{SB}}-v\left(k, a^{\mathrm{SB}}\right)-\frac{1}{2} r \sigma^{2} v_{a}^{2}\left(k, a^{\mathrm{SB}}\right),
\end{aligned}
$$

where the last equation refers to the joint surplus produced by the partnership under moral hazard. Note that the agent receives his reservation utility in both settings (i.e., there are no information rents). Furthermore, since utility is perfectly transferable, neither effort choice (be it first- or second-best) nor the strength of incentives (i.e., the power) depend on the agent's reservation utility. Instead, the fixed wage required to hire the agent is increasing in the reservation utility since the Participation Constraint is binding (Eq. 4). The second-best power of the incentive scheme is given by the marginal COE evaluated at the second-best effort (Eq. 3). By Eqs. (2) and (3), the second-best power depends directly and indirectly on the agent's ability, and only indirectly on the volatility of the project $\left(\sigma^{2}\right)$ and the agent's degree of risk aversion $(r)$ via the second-best exerted effort $a^{\mathrm{SB}}$.

\section{Analysis of the impact of ability in an isolated partnership}

It is important to start this section by highlighting the fact that a change in the value of the ability parameter affects both the total surplus and the ICC. ${ }^{15}$

\footnotetext{
14 The convexity of the COE function guarantees that the agent's certainty equivalent is concave in effort, and thus, that the First-Order Approach is valid in this setting.

15 In this way, the ability parameter's role is akin to that of the probability of an accident in Rothschild and Stiglitz (1976)'s imperfect-information insurance model, as said probability affects both the insurer's expected profits (objective function) and the insured's incentive and participation constraints.
} 
By the Envelope Theorem and Assumption 1, we have that:

$$
\begin{aligned}
& \frac{\partial \Pi^{\mathrm{FB}}}{\partial k}=-v_{k}\left(k, a^{\mathrm{FB}}\right)-\underline{\mathrm{CE}}^{\prime}(k) \\
& \frac{\partial \Pi^{\mathrm{SB}}}{\partial k}=-v_{k}\left(k, a^{\mathrm{SB}}\right)-r \sigma^{2} v_{a}\left(k, a^{\mathrm{SB}}\right) v_{a k}\left(k, a^{\mathrm{SB}}\right)-\underline{\mathrm{CE}^{\prime}}(k)
\end{aligned}
$$

If the reservation certainty-equivalent is not very sensitive to ability (i.e., $\mathrm{CE}$ is not too steep in $k$ if $\mathrm{CE}^{\prime}(k)<0$ ), the principal's expected profits are increasing in the agent's ability since the less able the agent, the more the principal must compensate him for: (i) his higher COE function (first term in Eqs. (7) and (8)); and (ii) his higher risk-premium since $v_{a k}>0$ (second term in Eq. 8). In such case, and independently of the risk of the project, the more talented the agent, the more desirable he is to the principal.

\subsection{Ability and effort}

The optimal contract induces a more talented agent to exert a strictly higher level of effort. This result is driven by the linearity of the production function, the strict convexity of the COE in effort and the supermodularity of the COE and the marginal COE functions in $a$ and $k .{ }^{16}$ By the linearity of the production function, the marginal cost of inducing an extra unit of effort (the left-hand side of Equations (1) and (2)) must remain constant in equilibrium. Said marginal cost is strictly decreasing in the agent's ability and strictly increasing in effort by Assumption 1, implying:

$$
\begin{aligned}
\frac{\mathrm{d} a^{\mathrm{FB}}}{\mathrm{d} k} & =-\frac{v_{a k}\left(k, a^{\mathrm{FB}}\right)}{v_{a a}\left(k, a^{\mathrm{FB}}\right)}<0 \\
\frac{\mathrm{d} a^{\mathrm{SB}}}{\mathrm{d} k} & =-\frac{v_{a k}\left(k, a^{\mathrm{SB}}\right)}{v_{a a}\left(k, a^{\mathrm{SB}}\right)}\left(1+\lambda\left(k, a^{\mathrm{SB}}\right)\right) \\
& =\underbrace{-\frac{v_{a k}\left(k, a^{\mathrm{SB}}\right)}{v_{a a}\left(k, a^{\mathrm{SB}}\right)}}_{\text {Direct Effect }(\mathrm{DE})} \underbrace{-\frac{v_{a k}\left(k, a^{\mathrm{SB}}\right)}{v_{a a}\left(k, a^{\mathrm{SB}}\right)} \lambda\left(k, a^{\mathrm{SB}}\right)}_{\text {Indirect Effect (IE) }}<0
\end{aligned}
$$

where

$$
\lambda(k, a):=\frac{\left(1-v_{a}(k, a)\right) \epsilon_{\frac{v_{a k}}{v_{a a}}, a}(k, a)}{\epsilon_{v_{a}, a}(k, a)+\left(1-v_{a}(k, a)\right) \epsilon_{v_{a a}, a}(k, a)}
$$

To understand the importance of the shape of the cost structure in analyzing the effect of ability on optimal effort, consider a diagram measuring the inverse of ability

\footnotetext{
${ }^{16}$ More broadly, the results also hold if the production function $f(a)$ is strictly concave in effort as then we just need to replace $v_{a}(k, a)$ with $v_{a}(k, a) / f_{a}(a)$ and $v_{a a}(k, a)$ with $\frac{\partial\left(v_{a}(k, a) / f_{a}(a)\right)}{\partial a}>0$ in Eqs. (1) and (2).
} 

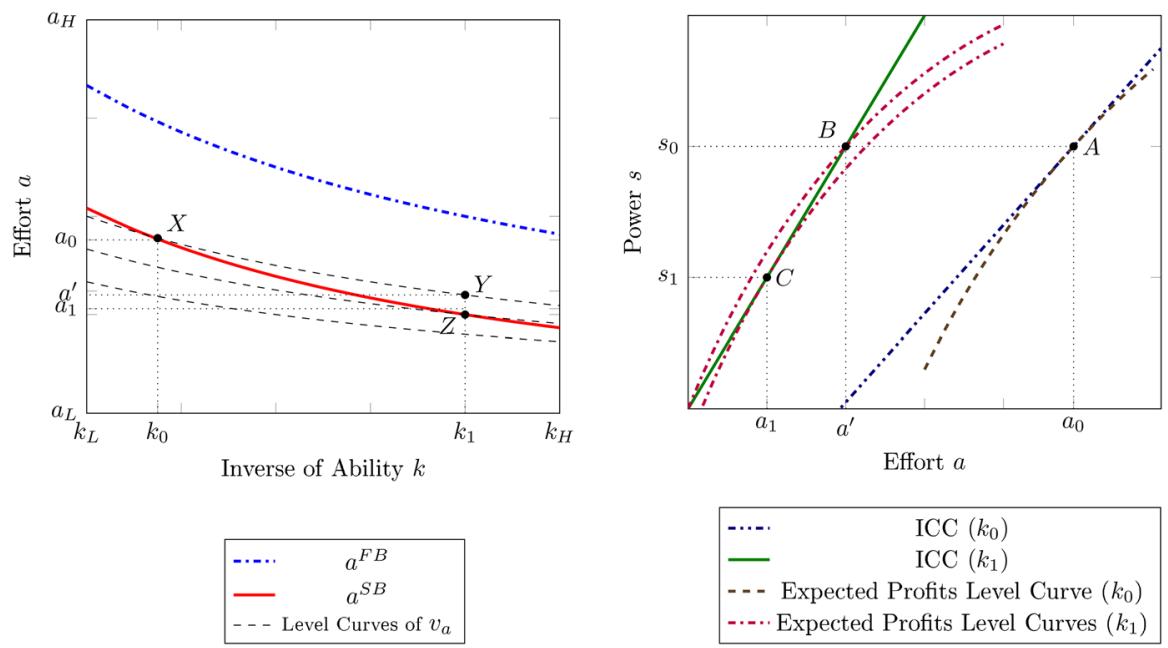

Fig. 1 Traditional scenario: the power increases with ability
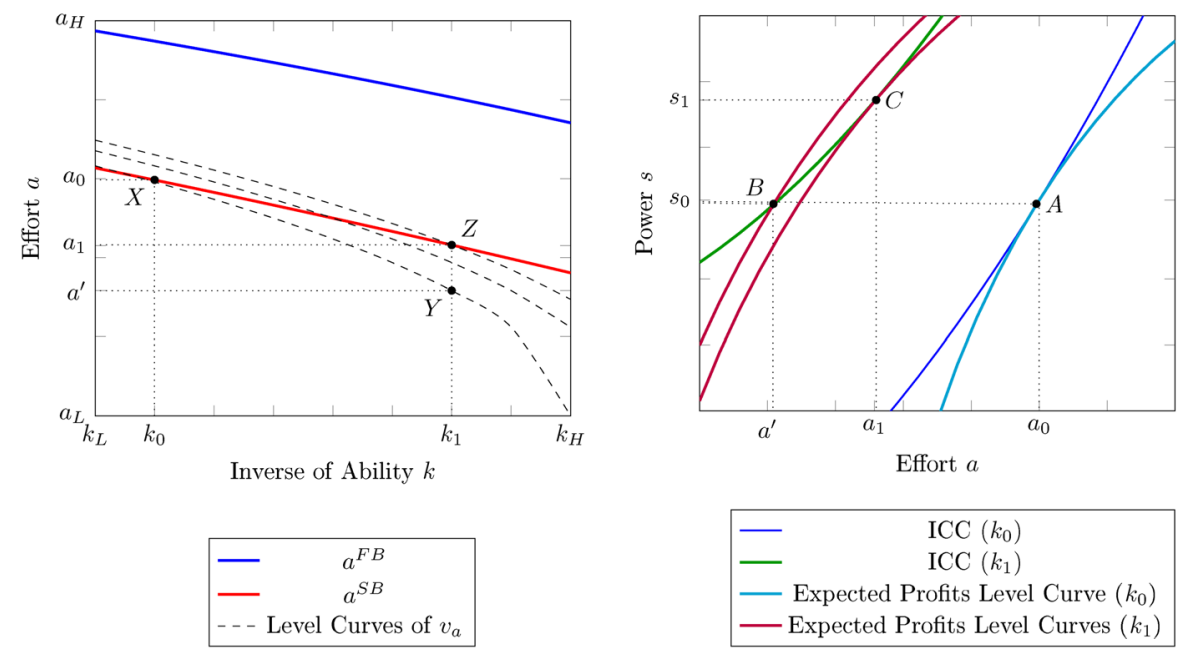

Fig. 2 Novel scenario: the power decreases with ability

$(k)$ in the horizontal axis and effort $(a)$ in the vertical axis, as illustrated in Figs. 1 and 2. The dashed lines in the left panels of Figs. 1 and 2 are the level curves of the marginal COE function. By Assumption 1, and for any given level of effort, a less able agent experiments a higher marginal disutility than a more able one. Hence, the level curves of the marginal COE are downward-slopping. The ratio $v_{a k} / v_{a a}$ is (the absolute value of) the marginal rate of substitution of the marginal COE, that is, the slope (in absolute values) of a level curve of the marginal COE: the steeper the level curve is, the lower the effort exerted by a less able agent must be-relative to a more able onefor both agents to experience the same marginal disutility of effort. Economically it reflects the relative sensitivity of said function to ability vis-a-vis effort, so that large 
(small) values (i.e., steeper (flatter) level curves) indicate that agents are (not) very responsive to changes in ability. Furthermore, in equilibrium-and as can be seen from Eq. (10) — it denotes the direct effect of ability on effort, for a fixed power of the incentive scheme: in other words, if the principal did not adjust the power of the incentive scheme to a change in the ability of the agent, the latter will adjust his effort choice by the magnitude of the marginal rate of substitution.

Equation (10) states that the total impact of ability on the second-best effort consists of two effects: (i) a direct effect (DE), which is given by the marginal rate of substitution of the marginal COE and reflects the response of the agent to a change in ability for $a$ given power $s$, and (ii) an indirect effect (IE) which is proportional to the bias $\lambda\left(k, a^{\mathrm{SB}}\right)$ (relative to the first-best) and embodies the principal's adjustment of the incentive scheme to said change in ability and the agent's behavioral response, reflecting the effect of the power on the risk-premium. ${ }^{17}$ Thus, the principal's response can either amplify, maintain or counteract the agent's direct response, depending on whether the bias is positive, equal to zero or negative. This can be seen graphically on the right panels of Figs. 1 and 2: the DE reflects the change in effort for given power, from initial point $\mathrm{A}$ to intermediate point $\mathrm{B}\left(a^{\prime}-a_{0}\right)$, while the IE corresponds to the shift from intermediate point $\mathrm{B}$ to final point $\mathrm{C}\left(a_{1}-a^{\prime}\right)$.

The assumption that less talented agents bear more convex disutilities than more able ones $\left(v_{a a k}(k, a) \geq 0\right.$ for all $\left.(k, a) \in K \times A\right)$ guarantees that $1+\lambda\left(k, a^{\mathrm{SB}}\right)>0$, which in turn implies that the equilibrium effort in the second-best scenario is increasing in the agent's ability. ${ }^{18}$ The sign of the bias is driven by the sign of $\epsilon \frac{v_{a k}}{v_{a a}}, a\left(k, a^{\mathrm{SB}}\right)$, that is, by the shape of the cost structure: its sign is positive if and only if the absolute value of the marginal rate of substitution is increasing in effort when evaluated at the second-best solution. ${ }^{19}$

Definition 1 For any given ability level $k$ : (i) the marginal COE satisfies the increasing marginal rate of substitution condition (IMRSC) at effort $\tilde{a}$ if the rate $v_{a k}(k, a) / v_{a a}(k, a)$ is strictly increasing in $a$ at $\tilde{a}$, that is, if $\partial\left(v_{a k}(k, \tilde{a}) / v_{a a}(k, \tilde{a})\right) / \partial a>$ 0 ; (ii) the marginal COE satisfies the diminishing marginal rate of substitution condition (DMRSC) at effort $\tilde{a}$ if the rate $v_{a k}(k, a) / v_{a a}(k, a)$ is strictly decreasing in $a$ at $\tilde{a}$, that is, if $\partial\left(v_{a k}(k, \tilde{a}) / v_{a a}(k, \tilde{a})\right) / \partial a<0$; the marginal COE satisfies the constant marginal rate of substitution condition (CMRSC) at effort $\tilde{a}$ if the rate $v_{a k}(k, a) / v_{a a}(k, a)$ is constant in $a$ at $\tilde{a}$, that is, if $\partial\left(v_{a k}(k, \tilde{a}) / v_{a a}(k, \tilde{a})\right) / \partial a=0$.

Note that Definition 1 involves partial (not total) derivatives with respect to variable a. Consider now a scenario in which the marginal $\mathrm{COE}$ is quasilinear with respect

17 "Appendix" provides further details of how Equations (10) and (11) are reached. As it is due to the risk premium, the equilibrium size of the bias is given by the sensitivity of the ratio $v_{a k} / v_{a a}$ to risk when evaluated at the second-best effort since $\lambda\left(k, a^{\mathrm{SB}}\right)=-\epsilon_{a^{\mathrm{SB}}, \sigma^{2}}\left(k, a^{\mathrm{SB}}\right) \epsilon \frac{v_{a k}}{v_{a a}}, a\left(k, a^{\mathrm{SB}}\right)$. Please, refer to Equation (19) located in "Appendix."

18 Furthermore, since $\epsilon_{\frac{v_{a k}}{v_{a a}}, a}(k, a)=\epsilon_{v_{a k}, a}(k, a)-\epsilon_{v_{a a}, a}(k, a)$ for all $(k, a) \in K \times A$, if the marginal COE is multiplicatively separable and $v_{a a a}>0$, as in the "canonical model," then $\epsilon_{v_{a k}, a}(k, a)=$ $\epsilon_{v_{a}, a}(k, a)$ for all $(k, a) \in K \times A$ which implies $\lambda\left(k, a^{\mathrm{SB}}\right)<1$ for all $k \in K$.

19 Equation (2) implies that $v_{a}\left(k, a^{\mathrm{SB}}\right)<1$, and together with the last equation in Assumption 1, it implies that the denominator of $\lambda(k, a \mathrm{SB})$ in Equation (11) is positive. 
to effort, so its level curves are vertically parallel for all parameter values. Then the marginal COE satisfies CMRSC for all parameter values implying a zero bias: $\epsilon \frac{v_{a k}, a}{v_{a a}}(k, a)=0$ for all $(k, a) \in K \times A$ implies $\lambda(k, a)=0$ for all $(k, a) \in K \times A$. In other words, for all values of $k$, the locus of all second-best effort choices coincides with a particular level curve of the marginal COE function and both the first-best and second-best efforts are equally responsive to ability.

There are two other special scenarios to consider: one in which the marginal COE satisfies the IMRSC for all parameter values, and another in which the marginal COE satisfies the DMRSC for all parameter values. For instance, take an homothetic marginal COE function. Then, if the superior sets were strictly convex, the secondbest effort curve (the solid line in the left panel of Fig. 1) would be steeper than the corresponding level curve of the marginal COE that passes through that combination of (ability, effort), implying a positive bias: the marginal COE would satisfy the IMRSC for all parameter values. In contrast, if the inferior sets were strictly convex, the second-best effort curve (the solid line in the left panel of Fig. 2) would be flatter than the corresponding level curve of the marginal COE that passes through that combination of (ability, effort) and the implied bias would be negative: the marginal COE would satisfy the DMRSC for all parameter values. Indeed, the responsiveness of the second-best effort to ability, relative to the slope of the corresponding marginal COE level curve, will be crucial when analyzing the total impact of ability on the strength of incentives.

For simplicity, in the next subsections, we will make some abuse of notation as we will refer to the first-best and second-best efforts simply as $a^{\mathrm{FB}}$ and $a^{\mathrm{SB}}$. The reader should keep in mind that they are functions of the agent's risk aversion level as well as the given characteristics of the participants in the relationship $\left(k, \sigma^{2}\right)$ : the agent's ability and the principal's project risk.

\subsection{Ability and incentives}

We remind the reader that the optimal power is given by the marginal COE evaluated at the second-best effort according to Eq. (3). An increase in the agent's ability causes two opposite effects on the optimal power of the incentive scheme: (i) a negative direct effect (first term in Equation (12) below) since more able agents are assumed to have lower marginal costs than their less able counterparts for any given effort level by Assumption 1; and (ii) a positive indirect effect via the adjustment of the second-best effort (second term in Equation (12)) since more able agents are induced to exert higher levels of effort optimally. The total effect of ability on the strength of incentives is driven by the balance of these two opposite forces:

$$
\frac{\mathrm{d} s^{\mathrm{SB}}}{\mathrm{d} k}=v_{a k}\left(k, a^{\mathrm{SB}}\right)+v_{a a}\left(k, a^{\mathrm{SB}}\right) \frac{\mathrm{d} a^{\mathrm{SB}}}{\mathrm{d} k}=-v_{a k}\left(k, a^{\mathrm{SB}}\right) \lambda\left(k, a^{\mathrm{SB}}\right),
$$

where the last equality follows from Equation (10).

Definition 2 Two basic scenarios can be distinguished depending on the sign of this relationship: 
(i) the "traditional" scenario, in which the optimal power is increasing in ability;

(ii) the "novel" scenario, in which the optimal power is decreasing in ability.

According to Eq. (12) and Definition 2, for any given ability level $k \in K$, the "traditional" ("novel") scenario occurs if and only if the bias is positive (negative).

Now, we turn to the main result of this section:

Proposition 1 Suppose Assumption 1 holds. For any given ability level, the optimal power $s \mathrm{SB}$ is strictly increasing (decreasing) in ability-i.e., the "traditional" ("novel") scenario occurs-if and only if the marginal COE satisfies the IMRSC (DMRSC) at $a^{\mathrm{SB}}$. The optimal power does not change with ability if and only if the marginal COE satisfies the CMRSC at $a^{\mathrm{SB}}$.

If the marginal COE satisfies the IMRSC at the second-best solution, then the second-best effort is sufficiently responsive to ability that the indirect effect of ability on the strength of incentives dominates overall in this case. In contrast, if the marginal COE satisfies the DMRSC at the second-best solution, then the optimal effort does not increase rapidly enough with ability and the direct effect of ability on incentives dominates. Finally, the total effect of ability on the strength of incentives is null if the marginal COE satisfies the CMRSC at $a^{\mathrm{SB}}$ as the two opposite effects exactly balance each other.

Figure 1 displays an example in which the marginal COE structure associated with the task satisfies the IMRSC for all parameter values implying a positive bias $(\lambda(k, a)>0$ for all $(k, a) \in K \times A)$. On the contrary, the marginal COE structure displayed in Fig. 2 satisfies the DMRSC for all parameter values implying a negative bias $(\lambda(k, a) \in(-1,0)$ for all $(k, a) \in K \times A)$.

To understand the intuition behind Proposition 1 , let us think of a principal who needs to hire a new agent who is less talented than his predecessor $\left(k_{1}>k_{0}\right)$. In the right panels of Figs. 1 and 2, the optimal power offered to the predecessor $\left(s_{0}\right)$ is determined by the point A, where the agent's incentive compatibility curve $\left(\operatorname{ICC}\left(k_{0}\right)\right)$ is tangent to the principal's expected profits level curve, being the tangency condition given by: $v_{a a}\left(k_{0}, a_{0}\right)=\left(1-v_{a}\left(k_{0}, a_{0}\right)\right) /\left(r \sigma^{2} s_{0}\right)$. Additionally, and according to Eq. (3), the power of the incentive scheme offered to the predecessor is implicitly represented by the level curve of the marginal $\mathrm{COE}$ associated with equilibrium point $\mathrm{X}$ in the left panels of Figs. 1 and 2. If the principal offered the new agent the same contract as his predecessor, the agent would optimally react by switching to point $\mathrm{B}$, exerting a lower level of effort $\left(a^{\prime}<a_{0}\right)$ according to his best response function-given by the $\operatorname{ICC}\left(k_{1}\right)$ curve in the right panels of Figs. 1 and 2-that is, $a^{\prime}$ solves $s_{0}=v_{a}\left(k_{1}, a^{\prime}\right)$. This implies a movement along the same level curve of the marginal COE (as the power does not vary) from point $\mathrm{X}$ to point $\mathrm{Y}$ in the left panels of Figs. 1 and 2. Such decrease in effort would generally not be optimal for the principal as the cost of providing incentives would generally change (i.e., $\left.v_{a a}\left(k_{1}, a^{\prime}\right) \neq v_{a a}\left(k_{0}, a_{0}\right)\right) .{ }^{20}$ In such case, the principal could sway the agent's behavior by adjusting the power of the

\footnotetext{
20 We use a discrete change in $k$ for ease of graphical exposition, but in reality, the inequality is satisfied for an infinitesimal change in $k$ : for any fixed power $s$ and ability level $k$, if $a^{\prime}(s, k)$ is defined by $v_{a}\left(k, a^{\prime}(s, k)\right) \equiv s$, then $\frac{\mathrm{d} v_{a a}\left(k, a^{\prime}(s, k)\right)}{\mathrm{d} k}=\frac{v_{a k}\left(k, a^{\prime}(s, k)\right)}{a^{\prime}(s, k)} \epsilon \frac{v_{a k}}{v_{a a}}, a\left(k, a^{\prime}(s, k)\right)$.
} 
scheme accordingly. The direction of said adjustment would depend on whether the principal wanted to intensify or counteract the agent's preliminary choice of effort $a^{\prime}$.

If the marginal COE structure satisfies the IMRSC, providing incentives to the new agent, relative to the old one, becomes more expensive than before since the $\operatorname{ICC}\left(k_{1}\right)$ curve is steeper than the $\operatorname{ICC}\left(k_{0}\right)$ curve at $a^{\prime}$ (i.e., $\left.v_{a a}\left(k_{1}, a^{\prime}\right)>v_{a a}\left(k_{0}, a_{0}\right)\right)$. Therefore, the principal wants to further decrease the agent's choice of effort by providing him weaker incentives. Notice that the new tangency condition is achieved at $a_{1}<a^{\prime}$ in the right panel of Fig. 1, leading to a strictly lower optimal power: $s_{1}=v_{a}\left(k_{1}, a_{1}\right)<v_{a}\left(k_{1}, a^{\prime}\right)=s_{0}$ and, hence, to the "traditional" scenario. Likewise, notice that $Z$ is below $Y$ in the left panel of Fig. 1. On the contrary, the provision of incentives to the new agent is cheaper than before under the DMRSC (i.e., $\left.v_{a a}\left(k_{1}, a^{\prime}\right)<v_{a a}\left(k_{0}, a_{0}\right)\right)$, and so the principal wants to induce the agent to exert more effort $\left(a_{1}>a^{\prime}\right)$ by providing him stronger incentives $\left(s_{1}>s_{0}\right.$, "novel" scenario) as it is illustrated in the right panel of Fig. 2. Notice also that $Z$ is above $Y$ in the left panel of Fig. 2. As a result, the same change in ability can lead to: (i) the "traditional" scenario if the marginal COE satisfies the IMRSC; or (ii) the "novel" scenario if the marginal COE satisfies the DMRSC.

A digression might be due at this point. As mentioned at the beginning of the subsection, the ability affects both the total surplus and the ICC. And this is the reason why - to determine the total effect of ability on the power of the scheme-we need to compare the slope of the total surplus' level curve and that of the ICC at point B (as we did in the previous two paragraphs and in the right panels of Figs. 1 and 2): they reflect-from the principal's point of view-the marginal benefit and marginal cost, respectively, of inducing an extra unit of effort from the agent via the adjustment of the power of the wage schedule. Yet, though this is indeed the economic intuition behind this exercise of comparative statics, we find that it can be expressed more compactly in terms of the MRS of the marginal COE function (as in Proposition 1). In turn, this means that the analysis based on the above-mentioned economic rationale (illustrated by the right panels of Figs. 1 and 2) is equivalent to the one based on the IMRSC/DMRSC property (and illustrated by the left panels of said figures). The left panels are thus a sort of closed form perspective that summarizes the actual analysis that the economic players carry out (right panels) when they face the "change-inability" comparative-statics case. The fact that the whole process can be distilled to a relatively simple technical condition on the COE function is a nice result of the model which helps us identify the two qualitatively different scenarios that can ensue (namely, the "traditional" and "novel" ones).

We proceed to illustrate our main result of the section. The optimal power under moral hazard is invariant to ability in Example 1 since the marginal COE satisfies the CMRSC for all parameter values.

Example 1 Assume $v(k, a)=k\left(\mathrm{e}^{\alpha a}-1\right)$, with $\alpha>0, A=[0,1], K \subset$ $\left(\left(\alpha \mathrm{e}^{\alpha}\right)^{-1},\left(\mathrm{e}^{\alpha}-1\right)^{-1}\right)$. This COE function satisfies Assumption 1. Since $v_{a k}(k, a) /$ $v_{a a}(k, a)=1 /(\alpha k)$ for all $(k, a) \in K \times A$, the marginal COE satisfies the CMRSC and the bias is zero for all parameter values. As a result, the second-best strength of incentives does not depend on the agent's ability level (e.g., $d s^{\mathrm{SB}} / d k=0$ ) by Proposition 1. 
In contrast, Example 2 illustrates a non-monotonic relationship between incentives and ability under moral hazard for some parameter values: if the project of the principal is of moderate risk and the parameter $\alpha$ is sufficiently high, the optimal power shows an inverted U-shape in ability since the marginal COE function satisfies the IMRSC (DMRSC) for sufficiently low (high) levels of effort, which are exerted by low- (high-) ability agents in equilibrium. Thus, the highest power is offered to an agent with an intermediate level of ability.

Example 2 Assume $v(k, a)=a^{2} k^{3}+(1 / \alpha) a^{\alpha}$, with $\alpha \geq 3 / 2,0=a_{L}<a_{H}<1$, and $\left(\frac{1-a_{H}^{\alpha-1}}{2 a_{H}}\right)^{\frac{1}{3}}<k_{L}<k_{H}<\left(\frac{\alpha-a_{H}^{\alpha-1}}{\alpha a_{H}}\right)^{\frac{1}{3}}$. This COE function satisfies Assumption 1. Furthermore, $\epsilon \frac{v_{a k}}{v_{a a}, a}(k, a)=\left(2 k^{3}+(\alpha-1)(3-\alpha) a^{\alpha-2}\right) /\left(2 k^{3}+(\alpha-1) a^{\alpha-2}\right)$ for all $(k, a) \in K \times A$. As a result, if $\alpha \in[3 / 2,3]$, then $\epsilon \frac{v_{a k}, a}{v_{a a}}(k, a)>0$ for all $(k, a) \in K \times A$ implying that the marginal COE satisfies the IMRSC for all $(k, a) \in K \times A$, and hence, the optimal power is increasing in ability by Proposition 1 . Suppose instead that $\alpha>3$. Then, the marginal COE satisfies the IMRSC (DMRSC) at $a$ if and only if $a<(>) \tilde{a}(k, \alpha)$ where $\tilde{a}(k, \alpha):=\left(2 k^{3} /((\alpha-1)(\alpha-3))\right)^{\frac{1}{\alpha-2}}$ is increasing in $k$. Let us further assume that $k_{L}<\bar{k}$ where $\bar{k}^{3}:=(1 / 2)(\alpha-1)(\alpha-3)(1+(\alpha-$ $1)(\alpha-3))^{-(\alpha-2) /(\alpha-1)}$, implying $a^{\mathrm{FB}}\left(k_{L}, \alpha\right)=a^{\mathrm{SB}}\left(k_{L}, r, 0, \alpha\right)>\tilde{a}\left(k_{L}, \alpha\right)$. Since $a^{\mathrm{SB}}$ is decreasing in $\sigma^{2}$, let us implicitly define $\sigma_{h}^{2}$ as $a^{\mathrm{SB}}\left(k_{L}, r, \sigma_{h}^{2}, \alpha\right) \equiv \tilde{a}\left(k_{L}, \alpha\right)$. Similarly, and by Eq. (10), $\sigma_{l}^{2}<\sigma_{h}^{2}$ satisfies $a^{\mathrm{SB}}\left(k_{H}, r, \sigma_{l}^{2}, \alpha\right) \equiv \tilde{a}\left(k_{H}, \alpha\right) .{ }^{21}$ Under moral hazard, if the principal's risk of the project is such that:

(i) $\sigma^{2} \leq \sigma_{l}^{2}$, then $\epsilon_{\frac{v_{a k}}{v_{a a}}, a}\left(k, a^{\mathrm{SB}}\right)<0$ for all $k \in\left[k_{L}, k_{H}\right)$ : the marginal COE satisfies the DMRSC at $a^{\mathrm{SB}}$, the bias is negative and the optimal power decreases with ability;

(ii) $\sigma_{l}^{2}<\sigma^{2}<\sigma_{h}^{2}$, then $\epsilon_{\frac{v_{a k}}{v_{a a}}, a}\left(k, a^{\mathrm{SB}}\right)<(>) 0$ for all $k<(>) \hat{k}$ where $\hat{k} \in K$ satisfies $a^{\mathrm{SB}}\left(\hat{k}, r, \sigma^{2}, \alpha\right) \equiv \tilde{a}(\hat{k}, \alpha)$ and it is decreasing in $\sigma^{2}$. Thus, the optimal power has an inverted U-shape in $k$ : the power decreases (increases) with ability for sufficiently (insufficiently) able agents;

(iii) $\sigma^{2} \geq \sigma_{h}^{2}$, then $\epsilon \frac{v_{a k}}{v_{a a}}, a\left(k, a^{\mathrm{SB}}\right)>0$ for all $k \in\left(k_{L}, k_{H}\right]$ : the marginal COE satisfies the IMRSC at $a^{\mathrm{SB}}$, the bias is positive and the optimal power increases with ability.

Ceteris paribus, the equilibrium relationship between incentives and ability is nonmonotonic if the principal's project is neither sufficiently risky nor sufficiently safe. In such case, an agent with an intermediate level of ability $(\hat{k})$ is offered the strongest incentives. If the principal's project is sufficiently risky, the optimal strength of incentives is monotonically increasing in ability, whereas if it is sufficiently safe, the optimal strength of incentives is monotonically decreasing in ability. In other words, this shows that the same COE function can generate some situations in which only the "traditional" scenario is observed for all parameter values (case iii), others in which only the "novel" one results for all parameter values (case i), and still others in which both occur, depending on the value of the parameters.

\footnotetext{
21 We remind the reader that Eq. (10) implies that the second-best effort in the second-best scenario is decreasing in $k$.
} 
The results obtained in Proposition 1 can be applied to common classes of cost structures, such as homothetic or separable marginal COEs. The following three Corollaries deal with these cases. Let first define $a^{\mathrm{FB}}\left(k_{L}\right)$ as the first-best effort level exerted by the most able agent (i.e., $\left.v_{a}\left(k_{L}, a^{\mathrm{FB}}\left(k_{L}\right)\right) \equiv 1\right)$.

Corollary 1 Let us suppose that Assumption 1 holds. If the marginal COE function is homothetic and strictly quasiconcave for all $(k, a) \in K \times\left[a^{\mathrm{SB}}\left(k_{H}, r, \sigma^{2}\right), a^{\mathrm{SB}}\left(k_{L}, r\right.\right.$, $\left.\sigma^{2}\right)$ ], then the equilibrium power $s^{\mathrm{SB}}$ strictly increases with ability. If the marginal COE function is homothetic and strictly quasiconvex for all $(k, a) \in K \times$ $\left[a^{\mathrm{SB}}\left(k_{H}, r, \sigma^{2}\right), a^{\mathrm{SB}}\left(k_{L}, r, \sigma^{2}\right)\right]$, then the equilibrium power ${ }^{\mathrm{SB}}$ strictly decreases with ability.

As mentioned in the previous subsection, if the marginal COE function is homothetic, then the IMRSC (DMRSC) holds for all parameter values if the superior (inferior) sets are strictly convex, that is, if the marginal COE function is strictly quasiconcave (quasiconvex). Examples 3 and 4 illustrate Corollary 1, generating a monotonic relationship between the optimal power and ability. Example 3 is a generalization of the "canonical model."

Example 3 Assume $v(k, a)=(1 / \alpha) a^{\alpha} k$ with $\alpha \geq 2, a_{L}=0<a_{H}$ and $a_{H}^{1-\alpha}<$ $k_{L}<k_{H} \leq \alpha a_{H}^{1-\alpha}$ (the "canonical model" corresponds to the case $\alpha=2$ ). This COE function satisfies Assumption 1 and it generates a marginal COE function that is homothetic and strictly quasiconcave for all $(k, a) \in K \times A$ since $v_{a k}(k, a) / v_{a a}(k, a)=(1 /(\alpha-1))(a / k)$ for all $(k, a) \in K \times A$, implying that the equilibrium power is strictly increasing in ability by Corollary 1 . Indeed, the marginal rate of substitution of the marginal COE function is unit-elastic in effort by Euler's Theorem: $\epsilon_{\frac{v_{a k}}{v_{a a}}, a}(k, a)=1$ for all $(k, a) \in K \times A$, and hence, the marginal COE satisfies the IMRSC for all parameter values.

Example 4 Assume $v(k, a)=\left(a^{\rho+1} /(\rho+1)\right)+a k^{\rho}$, with $\rho>1, a_{L}=0, a_{H} \in$ $(0,1),\left(1-a_{H}^{\rho}\right)^{1 / \rho}<k_{L}<k_{H} \leq\left(1-\left(a_{H}^{\rho} /(\rho+1)\right)\right)^{1 / \rho}$. This COE function satisfies Assumption 1. Since $v_{a k}(k, a) / v_{a a}(k, a)=(k / a)^{\rho-1}$, the marginal COE is homothetic and strictly quasiconvex for all $(k, a) \in K \times A$. Hence, the optimal power is strictly decreasing in ability by Corollary 1 . In fact, the marginal COE satisfies the DMRSC for all parameter values since $\epsilon \frac{v_{a k}}{v_{a a}}, a(k, a)=1-\rho<0$ for all $(k, a) \in K \times A$.

Under multiplicative separability of the marginal COE function, the IMRSC (DMRSC) holds for all parameter values if and only if the marginal COE function is log-concave (log-convex) in effort. We remind the reader that the marginal $\mathrm{COE}$ is log-concave (log-convex) in effort at any given level of effort $a$ if the ratio $v_{a}(k, a) / v_{a a}(k, a)$ is increasing (decreasing) in $a$.

Corollary 2 Let us suppose that Assumption1 holds and that the marginal COE function is multiplicatively separable. For any given ability level, the optimal power ${ }^{\mathrm{SB}}$ strictly increases (decreases) with ability if and only if the marginal COE is strictly logconcave (log-convex) in effort at $a^{\mathrm{SB}}$. 
Any function of effort which is homogeneous of degree $\alpha \geq 2$ (i.e., of the form $\left.f(a)=(1 / \alpha) a^{\alpha}\right)$ is log-concave in effort for all values of effort by Euler's Theorem. Thus, Corollary 2 and Euler's Theorem directly imply that the power is increasing in ability if the marginal COE function is multiplicatively separable and simultaneously homogeneous of degree $\alpha \geq 2$ in effort (as in Example 3 and the "canonical model") since then, $\epsilon \frac{v_{a k}}{v_{a a}, a}(k, a)=1>0$ for all $(k, a) \in K \times A$ by Euler's Theorem. Note that Corollary 2 can be applied to marginal COE functions that are not homothetic, as for instance, to a COE function of the form $v(k, a)=(1 / \alpha) a^{\alpha}\left(k^{\beta}+\gamma\right)$ with $\beta>1$ and any positive scalar $\gamma>0$. Corollary 2 implies that for IRMSC to hold for all parameter values, if the marginal COE function is multiplicative separable but not homothetic, then quasiconcavity must be replaced by a more restrictive condition: log-concavity in effort. ${ }^{22}$ According to Corollary 3, and relative to Corollary 2, even a stronger condition is required for the power to be monotonically increasing in ability under additive separability of the marginal COE, due to the absence of the interaction term. $^{23}$

Corollary 3 Let us suppose that Assumption 1 holds and that the marginal COE function is additively separable. For any given ability level, the optimal power $s^{\mathrm{SB}}$ strictly increases (decreases) with ability if and only if the marginal COE function is strictly concave (convex) in effort at $a^{\mathrm{SB}}$ and it is constant in ability if and only if the marginal COE function is linear in effort at $a^{\mathrm{SB}}$.

Note that the marginal COE displayed in Example 4 is simultaneously additively separable and strictly convex in effort for all parameter values since $\rho>1$. Thus, Corollary 3 can be applied, resulting in an incentive scheme strictly decreasing in ability.

In sum, the results displayed in this subsection emphasize the relevance of ability on the design of optimal compensation schemes and the importance of the IMRSC/DMRSC property of the marginal COE on shaping the second-best incentive scheme.

\subsection{Ability and performance pay}

For any given level of risk of the principal's project, more talented agents work harder than less talented ones in equilibrium. As a result, more talented agents unambiguously obtain a higher expected variable pay than less talented agents under the IMRSC or CMRSC since they are also offered stronger (or at least, no weaker) incentives. We now show that more talented agents may also obtain a higher variable pay under the DMRSC, i.e., when they are offered lower powered contracts than less talented agents.

Proposition 2 Let us suppose that Assumption 1 holds. For any given ability level, the expected performance pay $s^{\mathrm{SB}} a^{\mathrm{SB}}$ is strictly increasing (decreasing) in ability if and only if $\lambda\left(k, a^{\mathrm{SB}}\right)>(<)(-1)\left(1+\epsilon_{v_{a}, a}\left(k, a^{\mathrm{SB}}\right)\right)^{-1}$.

\footnotetext{
22 Recall that any log-concave function is quasi-concave.

23 Recall that concavity implies log-concavity.
} 
Proposition 2 states that more talented agents obtain a higher expected performance pay than less talented ones as long as the optimal effort is sufficiently responsive to ability, that is, as long as the bias is sufficiently high.

Example 5 Assume $v(k, a)=a k+\left(a^{3} / 3\right)$, with $a_{L}=0, a_{H} \in(0,1], 1-a_{H}^{2}<$ $k_{L}<k_{H} \leq 1-\left(a_{H}^{2} / 3\right)$. This COE function satisfies Assumption 1 and the marginal COE function satisfies the DMRSC for all parameter values since $\epsilon_{\frac{v_{a k}}{v_{a a}}, a}(k, a)=$ -1 for all $(k, a) \in K \times A$. Furthermore, $\epsilon_{v_{a a}, a}(k, a)=1$. These conditions imply that $\lambda(k, a)<0$ and $|\lambda(k, a)|=\left(1-v_{a}(k, a)+\epsilon_{v_{a}, a}(k, a)\right)^{-1}\left(1-v_{a}(k, a)\right)<$ $\left(1+\epsilon_{v_{a}, a}(k, a)\right)^{-1}$. Thus, although more talented agents are offered lower powered contracts, they work harder and obtain a higher expected variable pay than less talented agents by Proposition 2, since $\lambda(k, a)>(-1)\left(1+\epsilon_{v_{a}, a}(k, a)\right)^{-1}$ for all $(k, a) \in$ $K \times A .^{24}$

\subsection{Ability and efficiency loss}

The loss due to inefficient risk-sharing, also called "efficiency loss," is defined as $L\left(k, r, \sigma^{2}\right):=\Pi^{\mathrm{FB}}(k)-\Pi^{\mathrm{SB}}\left(k, r, \sigma^{2}\right)$. This section shows that the effect of ability on the efficiency loss is usually aligned with its effect on incentives.

By Eqs. (7) and (8),

$$
\frac{\partial L}{\partial k}=r \sigma^{2} v_{a}\left(k, a^{\mathrm{SB}}\right) v_{a k}\left(k, a^{\mathrm{SB}}\right)+\left[v_{k}\left(k, a^{\mathrm{SB}}\right)-v_{k}\left(k, a^{\mathrm{FB}}\right)\right]
$$

The first term in Equation (13) is positive and reflects the increase in the riskpremium that must be paid to a less able agent for the risk borne (the "risk-premium effect"). The second term in Equation (13) is negative and it reflects the impact of ability on the savings in wages paid in the second-best vis-a-vis the first-best (the "savings effect"): even if the risk premium is zero, any principal matched with a less talented agent must increase the compensation specified in the contract due to his higher disutility of effort if she wants the contract to be accepted. However, as a lower effort is implemented in equilibrium in the second-best than in the first-best (recall that the second-best effort choice is given by Eq. (2)) and $v_{a k}>0$ by Assumption 1 , the expected payment must be increased by less in the second-best relative to the first-best scenario, generating some savings for the principal. Proposition 3 specifies that the risk premium effect dominates the savings effect if and only if the marginal $\mathrm{COE}$ is relatively more convex than the function $v_{k}$ on $\left(a^{\mathrm{SB}}, a^{\mathrm{FB}}\right)$.

Proposition 3 Let us suppose that Assumption 1 holds. For any given ability level, the efficiency loss increases (decreases) with ability if and only if the function $v_{a}$ is relatively less (more) convex in effort than the function $v_{k}$ on $\left(a^{\mathrm{SB}}, a^{\mathrm{FB}}\right)$, that is, if and only if:

$$
v_{a k}\left(k, a^{\mathrm{SB}}\right) / v_{a a}\left(k, a^{\mathrm{SB}}\right) \leq(\geq)\left(v_{k}\left(k, a^{\mathrm{FB}}\right)-v_{k}\left(k, a^{\mathrm{SB}}\right)\right) /\left(v_{a}\left(k, a^{\mathrm{FB}}\right)-v_{a}\left(k, a^{\mathrm{SB}}\right)\right)
$$

\footnotetext{
24 Note that the marginal COE function is additively separable and convex in effort, so Corollary 3 can be applied in this example.
} 
The size of the risk premium effect depends on the curvature of the marginal COE by Equations (1) and (2): the more convex in effort the marginal COE is, the greater in size the risk premium is. A linear marginal $\mathrm{COE}$ implies a risk premium that is directly proportional to the effort distortion $a^{\mathrm{FB}}-a^{\mathrm{SB}}$. A convex (concave) marginal COE implies a risk premium that is more (less) than proportional to the effort distortion $a^{\mathrm{FB}}-a^{\mathrm{SB}}$. Analogously, the size of the savings effect depends on the curvature of the function $v_{k}$ : the more convex in effort the function $v_{k}$ is, the greater in size the savings effect is in absolute values. As a result, the risk premium effect dominates if and only if the marginal COE is relatively more convex in effort than the function $v_{k}$ on $\left(a^{\mathrm{SB}}, a^{\mathrm{FB}}\right)$. By Assumption $1, v_{k}(a, k)$ is assumed either linear or convex in effort for all parameter values. This assumption and Proposition 3 directly imply Corollary 4.

Corollary 4 Let us suppose that Assumption 1 holds. For any given ability level, if the marginal COE function is not convex in effort on $\left(a^{\mathrm{SB}}, a^{\mathrm{FB}}\right)$, the efficiency loss is increasing in ability.

For instance, if $\alpha \in[3 / 2,2)$, the marginal COE displayed in Example 2 is a strictly concave function of effort for all $(k, a) \in K \times A$. Thus, if $\alpha \in[3 / 2,2)$, the efficiency loss increases with ability in Example 2 by Corollary 4.

Furthermore, Proposition 3 implies Corollary 5 which provides a sufficient condition in terms of the marginal rate of substitution of the marginal COE for determining the sign of the relationship between the efficiency loss and ability.

Corollary 5 Let us suppose that Assumption 1 holds. For any given ability level, the efficiency loss increases (decreases) with ability if $v_{a k}\left(k, a^{\mathrm{SB}}\right) / v_{a a}\left(k, a^{\mathrm{SB}}\right) \leq(\geq$ )$v_{a k}(k, a) / v_{a a}(k, a)$ for all $a \in\left(a^{\mathrm{SB}}, a^{\mathrm{FB}}\right)$. For any given ability level, the efficiency loss is constant in ability if $v_{a k}(k, a) / v_{a a}(k, a)$ is invariant in effort on $\left(a^{\mathrm{SB}}, a^{\mathrm{FB}}\right)$.

For any given ability level $k$, the efficiency loss increases with ability if the marginal COE satisfies the IMRSC on $\left(a^{\mathrm{SB}}, a^{\mathrm{FB}}\right)$ by Corollary 5. Analogously, the satisfaction of the DMRSC on $\left(a^{\mathrm{SB}}, a^{\mathrm{FB}}\right)$ by the marginal COE is a sufficient condition for the efficiency loss to decrease with ability. We already showed that the marginal COE satisfies the IMRSC/CMRSC/DMRSC for all parameter values in Example 3 Examples 4 and 5. Therefore, the efficiency loss is increasing/constant/decreasing in ability in those examples, respectively, by Corollary 5 .

Corollaries 10, 11, and 12, located in Supplementary Appendix, are applications of Corollary 5 under different specifications of the marginal COE function and therefore, they are the analog of Corollaries 1,2 and 3.

\subsection{Ability and effort distortion}

In addition to the generation of a risk premium, the inefficient risk sharing also causes an effort distortion as a lower level of effort is exerted in the second-best scenario relative to the first-best. Let us define the effort distortion resulting from the information asymmetry as $D\left(k, r, \sigma^{2}\right):=a^{\mathrm{FB}}(k)-a^{\mathrm{SB}}\left(k, r, \sigma^{2}\right)$. The effort distortion decreases with ability if and only if the principal induces a more talented agent to increase the effort exerted in the second-best scenario by a higher amount than in the 
first-best scenario. The impact of ability on the effort distortion depends on the curvature of the marginal rate of substitution with respect to effort by Equations (9) and (10) and the fact that the equilibrium size of the bias is proportional to the rate of change of the marginal rate of substitution. Since the size of the equilibrium bias also depends on the sensitivity of the second-best effort to risk (recall that $\left.\lambda\left(k, a^{\mathrm{SB}}\right)=-\epsilon_{a^{\mathrm{SB}}, \sigma^{2}}\left(k, a^{\mathrm{SB}}\right) \epsilon \frac{v_{a k}, a}{v_{a a}}\left(k, a^{\mathrm{SB}}\right)\right)$, this sensitivity also plays a crucial role on determining the effect of ability on the effort distortion.

Proposition 4 Let us suppose that Assumption 1 holds. For any given ability level, effort underprovision increases (decreases) with ability if and only if:

$$
\frac{v_{a k}\left(k, a^{\mathrm{FB}}\right)}{v_{a a}\left(k, a^{\mathrm{FB}}\right)} \geq(\leq) \frac{v_{a k}\left(k, a^{\mathrm{SB}}\right)}{v_{a a}\left(k, a^{\mathrm{SB}}\right)}+\frac{\partial\left(\frac{v_{a k}\left(k, a^{\mathrm{SB}}\right)}{v_{a a}\left(k, a^{\mathrm{SB}}\right)}\right)}{\partial a} a^{\mathrm{SB}}\left(-\epsilon_{a^{\mathrm{SB}}, \sigma^{2}}\left(k, a^{\mathrm{SB}}\right)\right)
$$

It is immediate from Proposition 4 that if the marginal COE satisfies CMRSC for all parameter values as in Example 1, then effort underprovision is constant in ability. Suppose now that the marginal COE satisfies the IMRSC (DMRSC) at the second-best effort. Proposition 4 implies that for any given ability level, effort underprovision increases (decreases) with ability if the marginal rate of substitution function, $v_{a k}(k, a) / v_{a a}(k, a)$, is relatively convex in effort on $\left(a^{\mathrm{SB}}, a^{\mathrm{FB}}\right)$. To see this, suppose that the marginal rate of substitution is linearly increasing in effort for all parameter values (as in Example 3). Then, effort underprovision increases with ability if and only if the equilibrium size of the effort distortion is relatively high, that is, if and only if $a^{\mathrm{FB}}-a^{\mathrm{SB}} \geq a^{\mathrm{SB}}\left(-\epsilon_{a^{\mathrm{SB}}, \sigma^{2}}\left(k, a^{\mathrm{SB}}\right)\right)$ or equivalently, $1 \geq\left(1-\epsilon_{a^{\mathrm{SB}}, \sigma^{2}}\left(k, a^{\mathrm{SB}}\right)\right)\left(a^{\mathrm{SB}} / a^{\mathrm{FB}}\right)$. Note that this inequality is satisfied as long as the second-best effort is not too sensitive to risk, as is the case in the "canonical model" (and more broadly, in Example 3). In such cases, the power, the efficiency loss and the effort distortion move in unison as ability changes.

Example 6 Example 3 is revisited. By Eq. (19) located in "Appendix,"

$$
\epsilon_{a^{\mathrm{SB}}, \sigma^{2}}\left(k, a^{\mathrm{SB}}\right)=-\frac{\left(1-v_{a}\left(k, a^{\mathrm{SB}}\right)\right)}{\epsilon_{v_{a}, a}\left(k, a^{\mathrm{SB}}\right)+\left(1-v_{a}\left(k, a^{\mathrm{SB}}\right)\right) \epsilon_{v_{a a}, a}\left(k, a^{\mathrm{SB}}\right)}
$$

From Eq. (1), we have that:

$$
1-v_{a}\left(k, a^{\mathrm{SB}}\right)=1-\frac{v_{a}\left(k, a^{\mathrm{SB}}\right)}{v_{a}\left(k, a^{\mathrm{FB}}\right)}=1-\left(\frac{a^{\mathrm{SB}}}{a^{\mathrm{FB}}}\right)^{\alpha-1}
$$

Furthermore, $\epsilon_{v_{a}, a}\left(k, a^{\mathrm{SB}}\right)=\epsilon_{v_{a a}, a}\left(k, a^{\mathrm{SB}}\right)+1=\alpha-1$. Let us define $R:=$ $a^{\mathrm{SB}} / a^{\mathrm{FB}}<1$. Then

$$
\begin{aligned}
1-\epsilon_{a^{\mathrm{SB}}, \sigma^{2}}\left(k, a^{\mathrm{SB}}\right) & =1+\frac{\left(1-R^{\alpha-1}\right)}{(\alpha-1)+(\alpha-2)\left(1-R^{\alpha-1}\right)} \\
& =\frac{(\alpha-1)\left(2-R^{\alpha-1}\right)}{(\alpha-1)+(\alpha-2)\left(1-R^{\alpha-1}\right)}
\end{aligned}
$$


It is straightforward to see that $\left(1-\epsilon_{a^{\mathrm{SB}}, \sigma^{2}}\left(k, a^{\mathrm{SB}}\right)\right) R$ is increasing in $R$ and that $\lim _{R \rightarrow 1}\left(1-\epsilon_{a^{\mathrm{SB}}, \sigma^{2}}\left(k, a^{\mathrm{SB}}\right)\right) R=1$. Therefore, $1>\left(1-\epsilon_{a^{\mathrm{SB}}, \sigma^{2}}\left(k, a^{\mathrm{SB}}\right)\right)\left(a^{\mathrm{SB}} / a^{\mathrm{FB}}\right)$, and hence, the more talented the agent is, the greater the effort distortion is by Proposition 4.

The impact of ability on effort underprovision in Example 5 depends on the particular level of the agent's ability. In contrast with the "canonical model," the marginal rate of substitution of the marginal COE is inversely proportional to effort in Example 5, and hence, effort underprovision increases with ability if and only if the second-best effort is sufficiently sensitive to risk. ${ }^{25}$

Example 7 We revisit Example 5. Let us define $R:=a^{\mathrm{SB}} / a^{\mathrm{FB}}<1$. Notice that $\epsilon_{v_{a a}, a}\left(k, a^{\mathrm{SB}}\right)=1$ for all $k \in K$ and using Eq. (1):

$$
\begin{aligned}
1-v_{a}\left(k, a^{\mathrm{SB}}\right) & =1-\frac{v_{a}\left(k, a^{\mathrm{SB}}\right)}{v_{a}\left(k, a^{\mathrm{FB}}\right)}=\left(a^{\mathrm{FB}}\right)^{2}-\left(a^{\mathrm{SB}}\right)^{2}=\left(a^{\mathrm{FB}}\right)^{2}\left(1-R^{2}\right) \\
& =(1-k)\left(1-R^{2}\right)
\end{aligned}
$$

which implies

$$
\epsilon_{v_{a}, a}\left(k, a^{\mathrm{SB}}\right)=\frac{2\left(a^{\mathrm{SB}}\right)^{2}}{1-(1-k)\left(1-R^{2}\right)}
$$

and

$\epsilon_{a^{\mathrm{SB}}, \sigma^{2}}\left(k, a^{\mathrm{SB}}\right)=-\frac{1-R^{2}}{\left(\frac{2 R^{2}}{1-(1-k)\left(1-R^{2}\right)}\right)+\left(1-R^{2}\right)}=-\frac{1-R^{2}-(1-k)\left(1-R^{2}\right)^{2}}{1+R^{2}-(1-k)\left(1-R^{2}\right)^{2}}$

Then Eq. (14) becomes:

$$
0 \geq(\leq) \frac{1}{2 a^{\mathrm{SB}}}\left(1-R+\epsilon_{a^{\mathrm{SB}}, \sigma^{2}}\left(k, a^{\mathrm{SB}}\right)\right)
$$

Thus, effort underprovision increases (decreases) with ability if and only if $\left|\epsilon_{a^{\mathrm{SB}}, \sigma^{2}}\left(k, a^{\mathrm{SB}}\right)\right| \geq(\leq) 1-R$ or equivalently:

$$
k \geq(\leq) 1-(1+R)^{-2} \Leftrightarrow 1 \geq(\leq)\left(a^{\mathrm{FB}}+a^{\mathrm{SB}}\right)^{2}
$$

As both $a^{\mathrm{FB}}$ and $a^{\mathrm{SB}}$ are decreasing in $k$, the effort distortion decreases (increases) with ability if the agent is (is not) sufficiently talented. For instance, assume that $r \sigma^{2}=0.2, a_{H}=0.75$ and $K=[0.50,0.74]$. Then, the effort distortion is nonmonotonic in ability: it increases with $k$ (decreases with ability) for any $k \in[0.5,0.67]$ and it decreases with $k$ (increases with ability) for any $k \in[0.67,0.74]$. The left panel of Fig. 3 illustrates how the effort distortion reaches its highest value at an intermediate level of ability, $k=0.67$. 

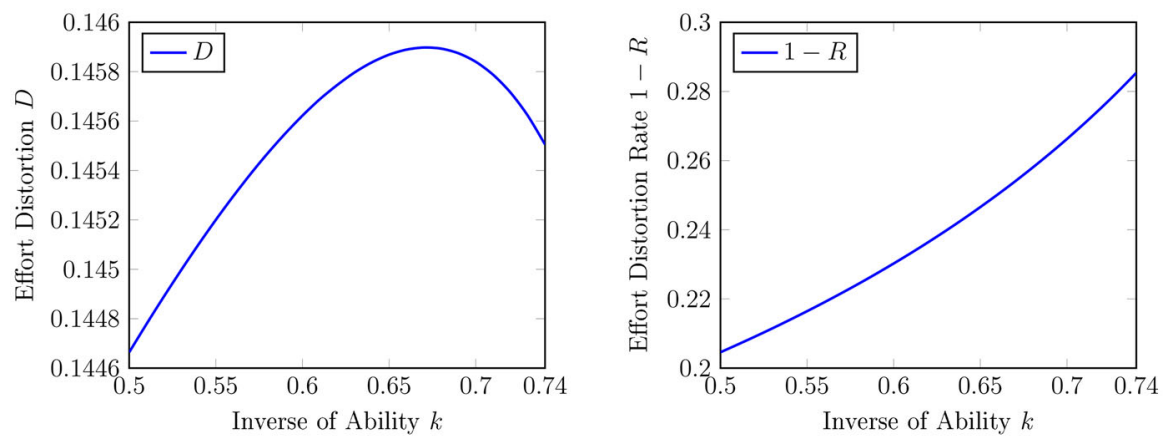

Fig. 3 Example 5 with $r \sigma^{2}=0.2, a_{H}=0.75$ and $K=[0.50,0.74]$. In the left panel, the effort distortion is non-monotonic in ability. In the right panel, the effort distortion rate is globally decreasing in ability

In sum, the power and the effort distortion need not move in unison after a shock in talent, as it has been illustrated in Example 7. As we already showed in Eq. (12), the direction of the impact of ability on the strength of incentives is driven by the sign of the bias (specifically, the sign of $\epsilon \frac{v_{a k}}{v_{a a}, a}\left(k, a^{\mathrm{SB}}\right)$ ), whereas the direction of the impact of ability on the effort distortion is driven by the size of the bias (specifically, the size of $\left.\left|\epsilon_{a^{\mathrm{SB}}, \sigma^{2}}\left(k, a^{\mathrm{SB}}\right)\right|\right)$.

We now briefly show that in some cases, like the canonical model, talent counteracts the negative effect of informational asymmetries on effort provision in percentage terms in spite of generating a greater effort distortion measured in absolute values. Let us formally define the effort-distortion rate as $1-R\left(k, r, \sigma^{2}\right)$ where $R\left(k, r, \sigma^{2}\right):=$ $a^{\mathrm{SB}}\left(k, r, \sigma^{2}\right) / a^{\mathrm{FB}}(k) \in(0,1)$ is the effort rate.

Proposition 5 Let us suppose that Assumption 1 holds. For any given ability level, the effort-distortion rate strictly decreases with ability if: $(i) \epsilon_{\frac{v_{a k}}{v_{a a}}, a}(k, a) \in[0,1]$ for all $a \in\left(a^{\mathrm{SB}}, a^{\mathrm{FB}}\right)$; or if (ii) the marginal $\mathrm{COE}$ is additively separable and of the form $v_{a}(k, a)=g(k)+f_{a}(a)$, where $f_{a}$ is an homogeneous of degree at least one function.

The effort rate increases with ability if and only if the principal induces a more talented agent to increase the effort exerted in the second-best scenario by

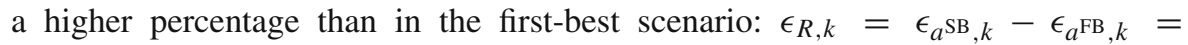
$\eta\left(k, a^{\mathrm{FB}}\right)-\eta\left(k, a^{\mathrm{SB}}\right)\left(1+\lambda\left(k, a^{\mathrm{SB}}\right)\right)$, where $\eta(k, a):=\left(v_{a k}(k, a) / v_{a a}(k, a)\right)(k / a)=$ $\epsilon_{v_{a}, k}(k, a) / \epsilon_{v_{a}, a}(k, a)$ for all $(k, a) \in K \times A$, by Eqs. (9) and (10). A non-negative but inelastic or unit-elastic marginal rate of substitution of the marginal COE with respect to effort for all parameter values (as in Example 3) guarantees a decreasing-in-ability effort-distortion rate for all levels of ability since it generates a non-negative bias in the second-best scenario and a decreasing-in-effort $\eta(k, a)$ function. Thus, if the marginal rate of substitution is not very sensitive to effort, we unambiguously obtain that the more talented the agent is, the lower the effort-distortion rate is.

A second sufficient condition for the effort-distortion rate to be strictly decreasing in ability is the specific form of additive separability of the marginal COE displayed

\footnotetext{
25 Note that in both examples, it is satisfied that $\left|\epsilon_{a^{\mathrm{SB}}, \sigma^{2}}\left(k, a^{\mathrm{SB}}\right)\right|<1$.
} 
in Proposition 5. For instance, Example 5 satisfies $\epsilon_{\frac{v_{a k}}{v_{a a}}, a}(k, a)=-1<0$ for all $(a, k) \in A \times K$, but Proposition 5 can still be applied since its marginal COE is given by $v_{a}(k, a)=k+a^{2}$. The right panel of Fig. 3 illustrates how the effort-distortion rate is strictly decreasing in ability in Example 5 despite its non-monotonicity when measured in absolute values (instead of percentage terms).

\section{Endogenous matching}

In Sects. 3 and 4, we analyzed the optimal contract in any given isolated relationship, that is, taking as given the characteristics of the participants in the relationship. As is well-known in the literature, by Equations (2) and (3), the higher the risk of the principal's project is, the lower the induced effort and the optimal power are in the second-best scenario. ${ }^{26}$ This is the negative direct effect of risk on optimal effort and incentives.

We now embed the principal-agent model in a competitive market setting to address the endogenous determination of the characteristics of the partnerships that sign contracts in the market. ${ }^{27}$ The characterization of the equilibrium matching between principals who differ in the risk of their projects and agents who differ in their ability is relevant as it has implications on agents' compensation. The results of this section constitute our second contribution to the agency literature: the equilibrium sorting pattern crucially depends on the structure of the COE function. Previous contributions which assumed heterogeneous-in-ability agents found a positive sorting in equilibrium under endogenous matching. Our contribution resides in identifying the conditions on the COE structure that lead to negative or positive sorting in equilibrium.

Let start our analysis by noting that the linear contract and the CARA riskpreferences turn the principal-agent model into a matching problem with perfectly transferable utility. Next, consider a competitive market where a unit-measure continuum of principals-who differ only in terms of the risk of their projects $\left(\sigma^{2} \in\right.$ $\left[\sigma_{L}^{2}, \sigma_{H}^{2}\right]:=\Sigma$ ) -endogenously match pairwise with a unit-measure continuum of agents, who differ only in their ability. ${ }^{28}$ Once a match between a principal of type $\sigma^{2}$ and an agent of type $k$ is formed, the equilibrium strength of incentives and effort choice are obtained as in Sect. $3 .^{29}$

We first focus on the conditions on the COE function that yield monotone assortative matching in equilibrium. If the agents' reservation certainty equivalent does not increase sharply with ability, the joint surplus of any given match is increasing in both the agent's ability and the firm's safety (i.e., decreasing in $k$ and $\sigma^{2}$ ) by the Envelope

\footnotetext{
26 Please, refer to Equation (18) located in "Appendix."

27 The book by Roth and Sotomayor (1990) is an excellent source for the theory of two-sided matching models.

28 Therefore, the COE function associated with the tasks to be executed by all agents is assumed to be the same across all matches. The matches are assumed to differ only in the traits of their partners.

29 Since utility is perfectly transferable, the fixed transfer from each principal to her agent will now be endogeneized in equilibrium and not be given by Eq. (4). Yet, the cost of effort and risk bearing must be added as in Eq. (4).
} 
Theorem. ${ }^{30}$ Define positive assortative matching, or PAM, as the case in which lowerrisk (lower- $\sigma^{2}$ ) principals match higher-ability (lower-k) agents. Similarly, negative assortative matching, or NAM, is the case in which lower-risk (lower- $\sigma^{2}$ ) principals match lower-ability (higher-k) agents.

Following Legros and Newman (2002)'s seminal paper, efficiency dictates PAM in equilibrium for all type distributions if and only if the equilibrium joint surplus is supermodular in $\left(k, \sigma^{2}\right)$ for all parameter values, that is, if and only if the traits of the agents and principals are complements (i.e., a riskier principal is less harmed by being matched with a less talented agent than a safer one). Analogously, there will be NAM in equilibrium for all type distributions if and only if the equilibrium joint surplus is submodular in $\left(k, \sigma^{2}\right)$ for all parameter values, that is, if and only if the traits of the agents and principals are substitutes for all parameter values (i.e., a riskier principal is more harmed by being matched with a less able agent than a safer one).

It turns out that the supermodularity/submodularity of the joint surplus is also driven by the sign of the bias.

Proposition 6 Let us suppose that Assumption 1 holds. In equilibrium, the economy is positively sorted for all type distributions if and only if the marginal COE does not satisfy the DMRSC for each $(k, a) \in K \times\left[a^{\mathrm{SB}}\left(k_{H}, r, \sigma_{H}^{2}\right), a^{\mathrm{SB}}\left(k_{L}, r, \sigma_{L}^{2}\right)\right]$. In equilibrium, the economy is negatively sorted for each type distributions if the marginal COE does not satisfy the IMRSC for each $(k, a) \in K \times\left[a^{\mathrm{SB}}\left(k_{H}, r, \sigma_{H}^{2}\right), a^{\mathrm{SB}}\left(k_{L}, r, \sigma_{L}^{2}\right)\right]$.

Proposition 6 resembles Proposition 1 since the joint surplus is supermodular if and only if the bias is positive (hence, the "traditional" scenario) and submodular if and only if the bias is negative (hence, the "novel" scenario). Therefore, PAM ensues in equilibrium in Example 3 since the marginal COE satisfies the IMRSC for all parameter values, whereas NAM ensues in equilibrium in Example 4 and Example 5 since the marginal COE satisfies the DMRSC for all parameter values. The equilibrium assignment in Example 1 could be either PAM and NAM as the marginal COE satisfies CMRSC for all parameter values.

The optimal equilibrium assignment in Example 2 depends on the principals' and/or agents' characteristics. If either all projects are sufficiently safe, or all the agents have a sufficiently low degree of risk aversion (i.e., if $\sigma_{H}^{2} \leq \sigma_{l}^{2}$ ), there is NAM in Example 2 since the bias is negative in equilibrium. If either all projects are sufficiently risky, or all the agents have a sufficiently high degree of risk aversion (i.e., if $\sigma_{L}^{2} \geq \sigma_{h}^{2}$ ), there is PAM in Example 2, as the bias is positive in equilibrium. On the contrary, if $\sigma_{L}^{2}<\sigma_{h}^{2}$ and $\sigma_{H}^{2}>\sigma_{l}^{2}$, the optimal assignment is not globally monotone assortative in Example 2 since the equilibrium matching is negatively assortative for all $\sigma^{2} \in$ $\left[\sigma_{L}^{2}, \sigma_{l}^{2}\right]$, but it is positively assortative for all $\sigma^{2} \in\left[\sigma_{h}^{2}, \sigma_{H}^{2}\right] .^{31}$

\footnotetext{
30 We remind the reader that if the agents' reservation certainty equivalent does not increase sharply with ability, then principals unanimously agree on the ranking of agents: a more talented agent is more desirable than a less talented one from all principals' perspectives by Eq. (8).

31 The possibly discontinuous equilibrium matching function could be fully characterized for specific numerical problems by solving the LP1 problem in the assignment game of Shapley and Shubik (1971), as done in Serfes (2008).
} 
The results stated in Proposition 6 can be suitably modified if the marginal COE is homothetic or separable. Thus, Corollaries 6, 7 and 8 are analogous to Corollaries 1, 2 and 3.

Corollary 6 Let us suppose that Assumption 1 holds. There is PAM in equilibrium for all type distributions if the marginal COE function is homothetic and strictly quasiconcave for all $(k, a) \in K \times\left[a^{\mathrm{SB}}\left(k_{H}, r, \sigma_{H}^{2}\right), a^{\mathrm{SB}}\left(k_{L}, r, \sigma_{L}^{2}\right)\right]$. Instead, there is NAM in equilibrium for all type distributions if the marginal COE function is homothetic and strictly quasiconvex for all $(k, a) \in K \times\left[a^{\mathrm{SB}}\left(k_{H}, r, \sigma_{H}^{2}\right), a^{\mathrm{SB}}\left(k_{L}, r, \sigma_{L}^{2}\right)\right]$.

Corollary 7 Let us suppose that Assumption 1 holds and that the marginal COE function is multiplicatively separable. Then, there is PAM in equilibrium for all type distributions if and only if the marginal COE is log-concave in effort for all $(k, a) \in K \times\left[a^{\mathrm{SB}}\left(k_{H}, r, \sigma_{H}^{2}\right), a^{\mathrm{SB}}\left(k_{L}, r, \sigma_{L}^{2}\right)\right]$. There is NAM in equilibrium for all type distributions if the marginal COE is log-convex in effort for all $(k, a) \in$ $K \times\left[a^{\mathrm{SB}}\left(k_{H}, r, \sigma_{H}^{2}\right), a^{\mathrm{SB}}\left(k_{L}, r, \sigma_{L}^{2}\right)\right]$.

Corollary 8 Let us suppose that Assumption 1 holds and that the marginal COE function is additively separable. There is PAM in equilibrium for all type distributions if and only if the marginal COE is concave in effort for all $(k, a) \in$ $K \times\left[a^{\mathrm{SB}}\left(k_{H}, r, \sigma_{H}^{2}\right), a^{\mathrm{SB}}\left(k_{L}, r, \sigma_{L}^{2}\right)\right]$ and there is $N A M$ in equilibrium for all type distributions if the marginal COE is convex in effort for all $(k, a) \in K \times$ $\left[a^{\mathrm{SB}}\left(k_{H}, r, \sigma_{H}^{2}\right), a^{\mathrm{SB}}\left(k_{L}, r, \sigma_{L}^{2}\right)\right]$.

Let $\mu\left(\sigma^{2}\right)$ denote the measure-preserving matching function under frictionless endogenous matching and let $\mu^{\prime}\left(\sigma^{2}\right)$ denote its derivative. The relationship between risk $\sigma^{2}$ and incentives $s^{\mathrm{SB}}$ is driven by two effects: the above-mentioned negative direct effect (the first term in Eq. (15)), implied by the CARA assumption, and an indirect effect (the second term in Eq. (15)), via the assignment. Specifically, the total effect of risk on incentives is given by:

$$
\begin{aligned}
\frac{\mathrm{d} s^{\mathrm{SB}}}{\mathrm{d} \sigma^{2}} & =v_{a a}\left(\mu\left(\sigma^{2}\right), a^{\mathrm{SB}}\right) \frac{\mathrm{d} a^{\mathrm{SB}}}{\mathrm{d} \sigma^{2}}+\left(v_{a a}\left(\mu\left(\sigma^{2}\right), a^{\mathrm{SB}}\right) \frac{\mathrm{d} a^{\mathrm{SB}}}{\mathrm{d} k}+v_{a k}\left(\mu\left(\sigma^{2}\right), a^{\mathrm{SB}}\right)\right) \mu^{\prime}\left(\sigma^{2}\right) \\
& =v_{a a}\left(\mu\left(\sigma^{2}\right), a^{\mathrm{SB}}\right)\left(\frac{\mathrm{d} a^{\mathrm{SB}}}{\mathrm{d} \sigma^{2}}-\frac{v_{a k}\left(\mu\left(\sigma^{2}\right), a^{\mathrm{SB}}\right)}{v_{a a}\left(\mu\left(\sigma^{2}\right), a^{\mathrm{SB}}\right)} \lambda\left(\mu\left(\sigma^{2}\right), a^{\mathrm{SB}}\right) \mu^{\prime}\left(\sigma^{2}\right)\right)
\end{aligned}
$$

where the second equality follows from Eq. (10) and $d a^{\mathrm{SB}} / d \sigma^{2}<0$ is computed in Eq. (18) in "Appendix." The indirect effect of risk on incentives (the second term inside the parenthesis of Eq. (16)) is proportional to the bias and negative under monotone assortative matching due to the perfect correlation between the sign of the bias and the monotone sorting pattern that ensues in equilibrium (Proposition 6). For any given ability level, the indirect effect of risk on incentives is: (i) zero if the marginal COE satisfies CMRSC for all parameter values, since the bias is zero; (ii) negative if the marginal COE satisfies the IMRSC for all parameter values since there is PAM by Proposition 6 and the bias is positive by Proposition 1; (iii) negative if the marginal 
COE satisfies the DMRSC for all parameter values since there is NAM by Proposition 6 and the bias is negative by Proposition 1. Corollary 9 summarizes these findings:

Corollary 9 Let us suppose that Assumption 1 holds. If the marginal COE does not satisfy the DMRSC for all $(k, a) \in K \times\left[a^{\mathrm{SB}}\left(k_{H}, r, \sigma_{H}^{2}\right), a^{\mathrm{SB}}\left(k_{L}, r, \sigma_{L}^{2}\right)\right]$, or alternatively, the marginal COE does not satisfy the IMRSC for all $(k, a) \in$ $K \times\left[a^{\mathrm{SB}}\left(k_{H}, r, \sigma_{H}^{2}\right), a^{\mathrm{SB}}\left(k_{L}, r, \sigma_{L}^{2}\right)\right]$, then the indirect effect of risk on incentives is non-positive for all type distributions, and therefore, the total effect of risk on incentives is negative.

Therefore, according to Corollary 9, under monotone assortative matching, the total effect of risk on incentives is unambiguously negative in equilibrium since the direct and indirect effects (if any) of risk on incentives reinforce each other. Intuitively, our model predicts that in an equilibrium characterized by monotone assortative matching, riskier principals sign lower-powered contracts since output is a relatively poor signal of effort, whereas safer principals sign higher-powered contracts since output is a relatively good signal of effort.

In Example 2, the optimal assignment is not globally monotone assortative for some parameter values. However, the indirect effect of risk on incentives is negative in all regions of the parameter space in which the matching function is locally continuous and monotone, since $\operatorname{sign}\left\{\mu^{\prime}\left(\sigma^{2}\right)\right\}=\operatorname{sign}\left\{\lambda\left(\mu\left(\sigma^{2}\right), a^{\mathrm{SB}}\left(\mu\left(\sigma^{2}\right), r, \sigma^{2}\right)\right)\right\}$ by the proof of Proposition 6. As a result, if the strength of incentives did not globally decrease with risk, it should be due to a discontinuity in the matching function.

The rest of the section analyzes the effect of risk on the equilibrium effort. As mentioned earlier, the direct effect of risk on the optimal effort is clearly negative. But there is also an indirect effect caused by the equilibrium assignment. If there is PAM in equilibrium (that is, if the marginal COE satisfies either the IMRSC or CMRSC for all parameter values), the direct and indirect effects of risk on effort reinforce each other. In equilibrium, riskier principals sign lower-powered contracts and attract less talented agents who exert a lower level of effort in equilibrium both due to their lower ability and to their higher exposition to risk. Suppose now that the marginal COE satisfies either the DMRSC or CMRSC for all parameter values and there is NAM in equilibrium by Proposition 6. In this case, the direct and indirect effects of risk on the optimal effort work in opposite directions since $\mathrm{d} a^{\mathrm{SB}} / \mathrm{d} \sigma^{2}<0$ but $\left(\mathrm{d} a^{\mathrm{SB}} / \mathrm{d} k\right) \mu^{\prime}\left(\sigma^{2}\right)>0$. In equilibrium, riskier principals sign lower-powered contracts but attract more talented agents. On the one hand, the higher ability of the agent induces the riskier principal to recommend a higher level of effort. On the other hand, the greater risk of her project induces the riskier principal to recommend a lower level of effort. The sign of the total effect of risk on optimal effort depends on which of these two effects dominates:

$$
\begin{aligned}
& \frac{\mathrm{d} a^{\mathrm{SB}}}{\mathrm{d} \sigma^{2}}+\frac{\mathrm{d} a^{\mathrm{SB}}}{\mathrm{d} k} \mu^{\prime}\left(\sigma^{2}\right) \geq(\leq) 0 \Leftrightarrow \\
& -\mu^{\prime}\left(\sigma^{2}\right) \geq(\leq) \frac{a^{\mathrm{SB}}}{\sigma^{2}} \frac{v_{a a}\left(\mu\left(\sigma^{2}\right), a^{\mathrm{SB}}\right)}{v_{a k}\left(\mu\left(\sigma^{2}\right), a^{\mathrm{SB}}\right)}\left(\frac{-\epsilon_{a^{\mathrm{SB}}, \sigma^{2}}\left(\mu\left(\sigma^{2}\right), a^{\mathrm{SB}}\right)}{1-\epsilon_{a} \mathrm{SB}, \sigma^{2}\left(\mu\left(\sigma^{2}\right), a^{\mathrm{SB}}\right) \epsilon_{\frac{v_{a}}{v_{a a}},}\left(\mu\left(\sigma^{2}\right), a^{\mathrm{SB}}\right)}\right) \Leftrightarrow \\
& -\mu^{\prime}\left(\sigma^{2}\right) \geq(\leq) \frac{a^{\mathrm{SB}}}{\sigma^{2}} \frac{v_{a a}\left(\mu\left(\sigma^{2}\right), a^{\mathrm{SB}}\right)}{v_{a k}\left(\mu\left(\sigma^{2}\right), a^{\mathrm{B}}\right)}\left(\frac{\left(1-v_{a}\left(\mu\left(\sigma^{2}\right), a^{\mathrm{SB}}\right)\right)}{\epsilon_{v_{a}, a}\left(\mu\left(\sigma^{2}\right), a^{\mathrm{SB}}\right)+\left(1-v_{a}\left(\mu\left(\sigma^{2}\right), a^{\mathrm{SB}}\right)\right) \epsilon_{v_{a k}, a}\left(\mu\left(\sigma^{2}\right), a^{\mathrm{SB}}\right)}\right)
\end{aligned}
$$


Our model predicts that in an equilibrium characterized by NAM, the indirect effect of risk on the optimal effort dominates and thus, more talented agents exert higher levels of effort than less talented ones if and only if the matching function is sufficiently steep. Alternatively, in an equilibrium characterized by NAM, the direct effect of risk on the optimal effort dominates and thus, more talented agents exert lower levels of effort than less talented ones if and only if the matching function is sufficiently flat.

\section{Conclusions}

Our paper highlights that incentives can depend on more dimensions than the principal's risk and the agent's risk aversion, such as the agent's ability and the nature of the task performed. We extend the existing theory, while encompassing previous models proposed in the agency literature. Specifically, we show the implications of generalizing the disutility of effort function under linear-CARA risk preferences. We find that, in equilibrium, there is a positive (negative) relationship between the power of the incentive scheme and the agent's ability - that is, we are in the "traditional" ("novel") scenario-if and only if the marginal COE satisfies the IMRSC (DMRSC).

The "canonical model" - in which the marginal COE function satisfies the IMRSC for all parameter values - is thus the quintessential example of the "traditional" scenario. Its two key predictions are that: (i) the strength of incentives is positively correlated with the ability of the agent and (ii) the stronger the incentives, the harder the agent will work. We show that these predictions do not hold true if the marginal COE satisfies the DMRSC since, in this case, high-ability agents face weaker incentives than low-ability ones but work harder in (partial) equilibrium.

Furthermore, we show that the market equilibrium sorting pattern is sensitive to those same properties of the marginal COE structure: the market is positively sorted in the "traditional" scenario (since the IMRSC holds for all parameter values) and negatively sorted in the "novel" scenario (since the DMRSC holds for all parameter values). Yet, the total effect of risk on incentives is negative under monotone assortative matching - that is, in both scenarios - since the indirect effect of risk on incentives reinforces the direct effect via the market assignment. Finally, the direct and indirect effect of risk on second-best effort reinforces each other only under positive assortative matching (i.e., in the "traditional" scenario). Under negative assortative matching (i.e., in the "novel" scenario), the direct (indirect) effect of risk on the optimal effort dominates if and only if the matching function is sufficiently flat (steep). In such case, efficiency dictates that riskier principals are matched with more talented agents, who are offered less powered contracts and exert lower (higher) levels of effort than less talented ones.

Modeling the heterogeneity of agents in talent and risk aversion and exploring the robustness of our results in a model with wealth effects are left for future research.

Acknowledgements We thank two anonymous referees and seminar participants from the Instituto de Investigaciones Economicas of the National University of Tucumán for their comments. 


\section{Declarations}

Conflict of interest The authors declare that they have no conflict of interest.

Ethical approval Both authors testify that this paper complies with the ethical rules of the journal and both agree with the submission of the paper to SERIEs. The authors declare that the work described has not been published before and that it is not under consideration for publication anywhere else. Finally, the authors declare no ethical issues or issues needing informed consent.

Open Access This article is licensed under a Creative Commons Attribution 4.0 International License, which permits use, sharing, adaptation, distribution and reproduction in any medium or format, as long as you give appropriate credit to the original author(s) and the source, provide a link to the Creative Commons licence, and indicate if changes were made. The images or other third party material in this article are included in the article's Creative Commons licence, unless indicated otherwise in a credit line to the material. If material is not included in the article's Creative Commons licence and your intended use is not permitted by statutory regulation or exceeds the permitted use, you will need to obtain permission directly from the copyright holder. To view a copy of this licence, visit http://creativecommons.org/licenses/by/4.0/.

\section{Appendix A: Proofs of results}

\section{Derivation of Equation (10):}

By Eq. (2) and the implicit function theorem:

$$
\begin{aligned}
\frac{\mathrm{d} a^{\mathrm{SB}}}{\mathrm{d} k} & =-\frac{v_{a k}\left(k, a^{\mathrm{SB}}\right)\left(1+r \sigma^{2} v_{a a}\left(k, a^{\mathrm{SB}}\right)\right)+r \sigma^{2} v_{a}\left(k, a^{\mathrm{SB}}\right) v_{a a k}\left(k, a^{\mathrm{SB}}\right)}{v_{a a}\left(k, a^{\mathrm{SB}}\right)\left(1+r \sigma^{2} v_{a a}\left(k, a^{\mathrm{SB}}\right)\right)+r \sigma^{2} v_{a}\left(k, a^{\mathrm{SB}}\right) v_{a a a}\left(k, a^{\mathrm{SB}}\right)}= \\
& =-\frac{v_{a k}\left(k, a^{\mathrm{SB}}\right)}{v_{a a}\left(k, a^{\mathrm{SB}}\right)}\left(\frac{1+r \sigma^{2} v_{a a}\left(k, a^{\mathrm{SB}}\right)+r \sigma^{2} v_{a}\left(k, a^{\mathrm{SB}}\right) \frac{\epsilon_{v_{a k}, a}\left(k, a^{\mathrm{SB}}\right)}{a}}{1+r \sigma^{2} v_{a a}\left(k, a^{\mathrm{SB}}\right)+r \sigma^{2} v_{a}\left(k, a^{\mathrm{SB}}\right) \frac{\epsilon_{v_{a a}, a}\left(k, a^{\mathrm{SB}}\right)}{a}}\right)= \\
& =-\frac{v_{a k}\left(k, a^{\mathrm{SB}}\right)}{v_{a a}\left(k, a^{\mathrm{SB}}\right)}\left(\frac{a+r \sigma^{2} v_{a}\left(k, a^{\mathrm{SB}}\right)\left(\epsilon_{v_{a}, a}\left(k, a^{\mathrm{SB}}\right)+\epsilon_{v_{a k}, a}\left(k, a^{\mathrm{SB}}\right)\right)}{a+r \sigma^{2} v_{a}\left(k, a^{\mathrm{SB}}\right)\left(\epsilon_{v_{a}, a}\left(k, a^{\mathrm{SB}}\right)+\epsilon_{v_{a a}, a}\left(k, a^{\mathrm{SB}}\right)\right)}\right)= \\
& =-\frac{v_{a k}\left(k, a^{\mathrm{SB}}\right)}{v_{a a}\left(k, a^{\mathrm{SB}}\right)}\left(1+\frac{r \sigma^{2} v_{a}\left(k, a^{\mathrm{SB}}\right)\left(\epsilon_{v_{a k}, a}\left(k, a^{\mathrm{SB}}\right)-\epsilon_{v_{a a}, a}\left(k, a^{\mathrm{SB}}\right)\right)}{a+r \sigma^{2} v_{a}\left(k, a^{\mathrm{SB}}\right)\left(\epsilon_{v_{a}, a}\left(k, a^{\mathrm{SB}}\right)+\epsilon_{v_{a a}, a}\left(k, a^{\mathrm{SB}}\right)\right)}\right)
\end{aligned}
$$

By Eq. (2), $r \sigma^{2} v_{a}\left(k, a^{\mathrm{SB}}\right)=\frac{1-v_{a}\left(k, a^{\mathrm{SB}}\right)}{v_{a a}\left(k, a^{\mathrm{SB}}\right)}$. Substituting this equality into the equation immediately above, multiplying and dividing the numerator and denominator by $v_{a a}\left(k, a^{\mathrm{SB}}\right) / v_{a}\left(k, a^{\mathrm{SB}}\right)$ and using Footnote (18), we get:

$$
\begin{aligned}
\frac{\mathrm{d} a^{\mathrm{SB}}}{\mathrm{d} k}= & -\frac{v_{a k}\left(k, a^{\mathrm{SB}}\right)}{v_{a a}\left(k, a^{\mathrm{SB}}\right)} \\
& \left(1+\frac{\left(\frac{1-v_{a}\left(k, a^{\mathrm{SB}}\right)}{v_{a}\left(k, a^{\mathrm{SB}}\right)}\right) \epsilon_{\frac{v_{a k}}{v_{a a}}, a}\left(k, a^{\mathrm{SB}}\right)}{\epsilon_{v_{a}, a}\left(k, a^{\mathrm{SB}}\right)+\left(\frac{1-v_{a}\left(k, a^{\mathrm{SB}}\right)}{v_{a}\left(k, a^{\mathrm{SB}}\right)}\right)\left(\epsilon_{v_{a}, a}\left(k, a^{\mathrm{SB}}\right)+\epsilon_{v_{a a}, a}\left(k, a^{\mathrm{SB}}\right)\right)}\right)
\end{aligned}
$$

Finally, Equation (10) is obtained by rearranging the denominator of the second term inside the parentheses. 
Proof of Proposition 1 The proof follows directly from Assumption 1 and Eqs. (11) and (12), since

$$
\epsilon \frac{v_{a k}, a}{v_{a a}}\left(k, a^{\mathrm{SB}}\right)=\frac{\partial\left(\frac{v_{a k}\left(k, a^{\mathrm{SB}}\right)}{v_{a a}\left(k, a^{\mathrm{SB}}\right)}\right)}{\partial a}\left(\frac{v_{a k}\left(k, a^{\mathrm{SB}}\right)}{v_{a a}\left(k, a^{\mathrm{SB}}\right)}\right)^{-1} a^{\mathrm{SB}} .
$$

Proof of Corollary 1 If the marginal COE is homothetic, then it is a monotonic transformation of an homogeneous-of-degree-one function: $v_{a}(k, a)=h(w(k, a))$ where $h(\cdot)$ is a strictly increasing function and $w(a, k)$ is homogeneous-of-degree-one. This implies that $w_{k}(k, a)$ and $w_{a}(k, a)$ are homogeneous of degree zero. As a result, for any scalar $t>0$, the marginal rate of substitution of the level curves of the marginal COE in a diagram with variable $k$ measured in the horizontal axis satisfies $\frac{v_{a k}(k, a)}{v_{a a}(k, a)}=\frac{w_{k}(k, a)}{w_{a}(k, a)}=\frac{w_{k}(t k, t a)}{w_{a}(t k, t a)}=\frac{v_{a k}(t k, t a)}{v_{a a}(t k, t a)}$. For any scalar $\alpha>1$, let us implicitly define $t>1$ by $v_{a}(k, \alpha a) \equiv v_{a}(t k, t a)$.

If the marginal COE is strictly quasiconcave for all $(k, a) \in K \times\left[a^{\mathrm{SB}}\left(k_{H}, r, \sigma^{2}\right)\right.$, $\left.a^{\mathrm{SB}}\left(k_{L}, r, \sigma^{2}\right)\right]$, then $\frac{v_{a k}(k, \alpha a)}{v_{a a}(k, \alpha a)}>\frac{v_{a k}(t k, t a)}{v_{a a}(t k, t a)}=\frac{v_{a k}(k, a)}{v_{a a}(k, a)}$ for all $(k, a) \in K \times$ $\left[a^{\mathrm{SB}}\left(k_{H}, r, \sigma^{2}\right), a^{\mathrm{SB}}\left(k_{L}, r, \sigma^{2}\right)\right]$. As $\frac{v_{a k}(k, a)}{v_{a a}(k, a)}$ is strictly increasing in $a$ for all $(k, a) \in$ $K \times\left[a^{\mathrm{SB}}\left(k_{H}, r, \sigma^{2}\right), a^{\mathrm{SB}}\left(k_{L}, r, \sigma^{2}\right)\right]$, then for any given $k \in K$, the marginal COE satisfies the IMRSC at $a^{\mathrm{SB}}$. The result follows by applying Proposition 1 .

Conversely, if the marginal COE is strictly quasiconvex for all $(k, a) \in K \times$ $\left[a^{\mathrm{SB}}\left(k_{H}, r, \sigma^{2}\right), a^{\mathrm{SB}}\left(k_{L}, r, \sigma^{2}\right)\right]$, then $\frac{v_{a k}(k, \alpha a)}{v_{a a}(k, \alpha a)}<\frac{v_{a k}(t k, t a)}{v_{a a}(t k, t a)}=\frac{v_{a k}(k, a)}{v_{a a}(k, a)}$ for all $(k, a) \in$ $K \times\left[a^{\mathrm{SB}}\left(k_{H}, r, \sigma^{2}\right), a^{\mathrm{SB}}\left(k_{L}, r, \sigma^{2}\right)\right]$. Thus, for any given $k \in K$, the marginal COE satisfies the DMRSC at $a^{\mathrm{SB}}$. The result follows by applying Proposition 1 .

Proof of Corollary 2 If the marginal COE is multiplicatively separable, then it is of the form $v_{a}(k, a)=g(k) f_{a}(a)$. Hence, $v_{a k}(k, a) / v_{a a}(k, a)=\left(g^{\prime}(k) / g(k)\right)\left(f_{a}(a) / f_{a a}(a)\right)$.

If the marginal COE is strictly log-concave in effort at $a^{\mathrm{SB}}$, then $f_{a}$ is strictly $\log$-concave in $a$ at $a^{\mathrm{SB}}$ since $v_{a}(k, a) / v_{a a}(k, a)=f_{a}(a) / f_{a a}(a)$. Since $f_{a}$ is strictly $\log$-concave in $a$ at $a^{\mathrm{SB}}, f_{a}(a) / f_{a a}(a)$ is strictly increasing in $a$ at $a^{\mathrm{SB}}$ implying $\epsilon \frac{v_{a k}, a}{v_{a a}}\left(k, a^{\mathrm{SB}}\right)>0$ for all $k \in K$. Hence, $\lambda\left(k, a^{\mathrm{SB}}\right)>0$ for all $k \in K$ and $\mathrm{d} s \mathrm{SB} / \mathrm{d} k<0$ by Eq. (12). For the necessary result, by Eq. (12), $\mathrm{d} s \mathrm{SB} / \mathrm{d} k<0$ only if $\lambda\left(k, a^{\mathrm{SB}}\right)>0$ for all $k \in K$. By Eqs. (2) and (11) and Assumption 1, $\lambda\left(k, a^{\mathrm{SB}}\right)>0$ for all $k \in K$ only if $\epsilon_{\frac{v_{a k}}{v_{a}}, a}\left(k, a^{\mathrm{SB}}\right)>0$ for all $k \in K$. Since $v_{a k}(k, a) / v_{a a}(k, a)=\left(g^{\prime}(k) / g(k)\right)\left(f_{a}(a) / f_{a a}(a)\right), \epsilon \frac{v_{a k}, a}{v_{a a}}\left(k, a^{\mathrm{SB}}\right)>0$ for all $k \in K$ only if $f_{a}(a) / f_{a a}(a)$ is strictly increasing in $a$ at $a^{\mathrm{SB}}$, that is, only if $v_{a}(k, a) / v_{a a}(k, a)$ is strictly increasing in $a$ at $a^{\mathrm{SB}}$, or equivalently, $v_{a}$ is strictly log-concave in $a$ at $a^{\mathrm{SB}}$.

The procedure is analogous for the log-convexity case.

Proof of Corollary 3 Let us assume that the marginal COE satisfies Assumption 1 and it is of the form $v_{a}(k, a)=g(k)+f_{a}(a)$ for all $(k, a) \in K \times A$. Then $\frac{v_{a k}(k, a)}{v_{a a}(k, a)}=\frac{g^{\prime}(k)}{f_{a a}(a)}$. The result follows by directly applying Proposition 1. 
Proof of Proposition 2 By Eqs. (3) and (10), we get that:

$$
\begin{aligned}
\frac{\mathrm{d} s^{\mathrm{SB}} a^{\mathrm{SB}}}{\mathrm{d} k} & =v_{a k}\left(k, a^{\mathrm{SB}}\right) a^{\mathrm{SB}}+\left(v_{a a}\left(k, a^{\mathrm{SB}}\right) a^{\mathrm{SB}}+v_{a}\left(k, a^{\mathrm{SB}}\right)\right) \frac{\mathrm{d} a^{\mathrm{SB}}}{\mathrm{d} k}= \\
& =\left(-\frac{v_{a k}\left(k, a^{\mathrm{SB}}\right)}{v_{a a}\left(k, a^{\mathrm{SB}}\right)}\right) v_{a}\left(k, a^{\mathrm{SB}}\right)\left(1+\lambda\left(k, a^{\mathrm{SB}}\right)\left(1+\epsilon_{v_{a}, a}\left(k, a^{\mathrm{SB}}\right)\right)\right)
\end{aligned}
$$

Therefore, by Assumption $1, \mathrm{~d}\left(s^{\mathrm{SB}} a^{\mathrm{SB}}\right) / \mathrm{d} k<(>) 0$ if and only if $1+\lambda\left(k, a^{\mathrm{SB}}\right)(1+$ $\left.\epsilon_{v_{a}, a}\left(k, a^{\mathrm{SB}}\right)\right)>(<) 0$ or equivalently, $\lambda\left(k, a^{\mathrm{SB}}\right)>(<)-\left(1+\epsilon_{v_{a}, a}\left(k, a^{\mathrm{SB}}\right)\right)^{-1}$.

Proof of Proposition 3 By the use of Eqs. (1) and (2), Eq. (13) can be rewritten as follows:

$$
\frac{\partial L}{\partial k}=v_{a k}\left(k, a^{\mathrm{SB}}\right)\left[\left(\frac{v_{a}\left(k, a^{\mathrm{FB}}\right)-v_{a}\left(k, a^{\mathrm{SB}}\right)}{v_{a a}\left(k, a^{\mathrm{SB}}\right)}\right)-\left(\frac{v_{k}\left(k, a^{\mathrm{FB}}\right)-v_{k}\left(k, a^{\mathrm{SB}}\right)}{v_{a k}\left(k, a^{\mathrm{SB}}\right)}\right)\right]
$$

The result is obtained by rearranging Eq. (17).

Proof of Corollary 4 First, we show that Assumption 1 implies that $v_{k}$ is either linear or convex in effort for all $a \in\left(a^{\mathrm{SB}}, a^{\mathrm{FB}}\right)$. To see this, notice that $v_{a a k}(k, a) \geq 0$ for all $(k, a) \in K \times A$ such that $a>a_{L}$ implies that $\frac{v_{k}\left(k, a^{\mathrm{FB}}\right)-v_{k}\left(k, a^{\mathrm{SB}}\right)}{v_{a k}\left(k, a^{\mathrm{SB}}\right)}=\int_{a^{\mathrm{SB}}}^{a^{\mathrm{FB}}} \frac{v_{a k}(k, a)}{v_{a k}\left(k, a^{\mathrm{SB}}\right)} \mathrm{d} a \geq$ $\int_{a^{\mathrm{SB}}}^{a^{\mathrm{FB}}} 1 \mathrm{~d} a=a^{\mathrm{FB}}-a^{\mathrm{SB}}$ for any given $k \in K$, and hence, $v_{k}\left(k, a^{\mathrm{FB}}\right) \geq v_{k}\left(k, a^{\mathrm{SB}}\right)+$ $v_{a k}\left(k, a^{\mathrm{SB}}\right)\left(a^{\mathrm{FB}}-a^{\mathrm{SB}}\right)$ for any given $k \in K$. Fix $k \in K$. If $v_{a}$ is not convex in effort on $\left(a^{\mathrm{SB}}, a^{\mathrm{FB}}\right)$, then $v_{a}\left(k, a^{\mathrm{FB}}\right) \leq v_{a}\left(k, a^{\mathrm{SB}}\right)+v_{a a}\left(k, a^{\mathrm{SB}}\right)\left(a^{\mathrm{FB}}-a^{\mathrm{SB}}\right)$, implying $\frac{v_{a}\left(k, a^{\mathrm{FB}}\right)-v_{a}\left(k, a^{\mathrm{SB}}\right)}{v_{a a}\left(k, a^{\mathrm{SB}}\right)} \leq a^{\mathrm{FB}}-a^{\mathrm{SB}} \leq \frac{v_{k}\left(k, a^{\mathrm{FB}}\right)-v_{k}\left(k, a^{\mathrm{SB}}\right)}{v_{a k}\left(k, a^{\mathrm{SB}}\right)}$. By applying Eq. (17), we get that $\frac{\partial L}{\partial k} \leq 0$.

Proof of Corollary 5 Fix $k \in K$. By rearranging Eq. (17), we get that $\frac{\partial L}{\partial k} \geq(\leq) 0$ if and only if:

$$
\begin{gathered}
\int_{a^{\mathrm{SB}}}^{a^{\mathrm{FB}}} v_{a a}(k, a) \mathrm{d} a \geq(\leq) \frac{v_{a a}\left(k, a^{\mathrm{SB}}\right)}{v_{a k}\left(k, a^{\mathrm{SB}}\right)} \int_{a^{\mathrm{SB}}}^{a^{\mathrm{FB}}} v_{a k}(k, a) \mathrm{d} a \\
\Longleftrightarrow \int_{a^{\mathrm{SB}}}^{a^{\mathrm{FB}}}\left(\frac{v_{a a}(k, a)}{v_{a k}(k, a)}-\frac{v_{a a}\left(k, a^{\mathrm{SB}}\right)}{v_{a k}\left(k, a^{\mathrm{SB}}\right)}\right) v_{a k}(k, a) \mathrm{d} a \geq(\leq) 0
\end{gathered}
$$


If $\frac{v_{a k}(k, a)}{v_{a a}(k, a)} \geq \frac{v_{a k}\left(k, a^{\mathrm{SB}}\right)}{v_{a a}\left(k, a^{\mathrm{SB}}\right)}$ for all $a \in\left(a^{\mathrm{SB}}, a^{\mathrm{FB}}\right)$, then $\frac{\partial L}{\partial k} \leq 0$. Analogously, if $\frac{v_{a k}(k, a)}{v_{a a}(k, a)} \leq$ $\frac{v_{a k}\left(k, a^{\mathrm{SB}}\right)}{v_{a a}\left(k, a^{\mathrm{SB}}\right)}$ for all $a \in\left(a^{\mathrm{SB}}, a^{\mathrm{FB}}\right)$, then $\frac{\partial L}{\partial k} \geq 0$.

Proof of Proposition 4 By Eqs. (9) and (10):

$$
\frac{\mathrm{d} D}{\mathrm{~d} k}=\frac{\mathrm{d} a^{\mathrm{FB}}}{\mathrm{d} k}-\frac{\mathrm{d} a^{\mathrm{SB}}}{\mathrm{d} k}=-\frac{v_{a k}\left(k, a^{\mathrm{FB}}\right)}{v_{a a}\left(k, a^{\mathrm{FB}}\right)}+\frac{v_{a k}\left(k, a^{\mathrm{SB}}\right)}{v_{a a}\left(k, a^{\mathrm{SB}}\right)}\left(1+\lambda\left(k, a^{\mathrm{SB}}\right)\right)
$$

Since $\lambda\left(k, a^{\mathrm{SB}}\right)=-\epsilon_{a^{\mathrm{SB}}, \sigma^{2}}\left(k, a^{\mathrm{SB}}\right) \epsilon \frac{v_{a k}}{v_{a a}, a}\left(k, a^{\mathrm{SB}}\right)$, the result is obtained by substituting this expression into the above equation.

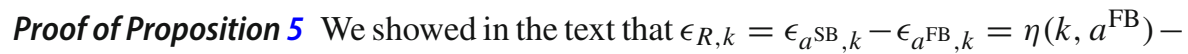
$\eta\left(k, a^{\mathrm{SB}}\right)\left(1+\lambda\left(k, a^{\mathrm{SB}}\right)\right)$.

The first sufficient condition in Proposition 5 follows directly from the fact that $\epsilon_{\eta, a}(k, a)=\epsilon_{\frac{v_{a k}}{v_{a a}}, a}(k, a)-1$.

If the marginal COE is additively separable, i.e., $v_{a}(k, a)=g(k)+f_{a}(a)$, then $v_{a k}(k, a)=g^{\prime}(k)$ and $v_{a a}(k, a)=f_{a a}(a)$ for all $(k, a) \in K \times A$. Hence, $\eta(k, a)=$ $\left(k g^{\prime}(k) / a f_{a a}(a)\right)$. In addition, $\epsilon_{v_{a k}, a}(k, a)=0$ and $\epsilon_{v_{a a}, a}(k, a)=\epsilon_{f_{a a}, a}(a)$ implying $\epsilon_{\frac{v_{a k}}{v_{a a}}, a}(k, a)=-\epsilon_{f_{a a}, a}(a)$ for all $(k, a) \in K \times A$. By Eq. $(11), \eta(k, a)(1+\lambda(k, a))=$ $k g^{\prime}(k) / \psi(k, a)$ where $\psi(k, a):=a f_{a a}(a)+v_{a}(k, a)\left(1-v_{a}(k, a)\right) \epsilon_{f_{a a}, a}(a)$. The homogeneity of degree $n$ implies - by Euler's theorem-that $\epsilon_{f_{a}, a}(a)$ is a constant (and henceforth, it will be denoted by $\epsilon_{f_{a}, a}$ ) equal to its degree of homogeneity and $\epsilon_{f_{a}, a}=\epsilon_{f_{a a}, a}+1$. Since $\epsilon_{f_{a a}, a}$ is also a constant, then $\frac{\partial \epsilon_{f a a} a}{\partial a}=0$. This, in turn, implies that

$$
\frac{\partial \psi(k, a)}{\partial a}=f_{a a}(a)\left(1+2 \epsilon_{f_{a a}, a}\left(1-v_{a}(k, a)\right)\right)
$$

Fix $k \in K$. If $\epsilon_{f_{a}, a} \geq 1$, then $\epsilon_{f_{a a}, a}=\epsilon_{f_{a}, a}-1 \geq 0$, and by Assumption 1 and Eq. (2), $\frac{\partial \psi(k, a)}{\partial a}>0$ for all $a \in\left[a_{L}, a^{\mathrm{FB}}(k)\right]$. In turn, this implies that $\eta(k, a)(1+\lambda(k, a))$ is strictly decreasing in $a$ for all $a \in\left[a_{L}, a^{\mathrm{FB}}(k)\right]$.

Proof of Proposition 6 By applying the Envelope Theorem to Eq. (6), we find that the joint surplus is increasing in both the principal's safety and the agent's ability. As it is well known in the literature (please refer to Legros and Newman (2002), supermodularity (submodularity) of the joint surplus is a necessary and sufficient condition for PAM (NAM) for all distributions of agents' and principals' types. From Eq. (2) and Assumption 1,

$$
\frac{\mathrm{d} a^{\mathrm{SB}}}{\mathrm{d} \sigma^{2}}=-\frac{r v_{a}\left(k, a^{\mathrm{SB}}\right) v_{a a}\left(k, a^{\mathrm{SB}}\right)}{v_{a a}\left(k, a^{\mathrm{SB}}\right)+r \sigma^{2}\left(v_{a a}^{2}\left(k, a^{\mathrm{SB}}\right)+v_{a}\left(k, a^{\mathrm{SB}}\right) v_{a a a}\left(k, a^{\mathrm{SB}}\right)\right)}=
$$




$$
=-\frac{a^{\mathrm{SB}}}{\sigma^{2}} \frac{\left(1-v_{a}\left(k, a^{\mathrm{SB}}\right)\right)}{\epsilon_{v_{a}, a}\left(k, a^{\mathrm{SB}}\right)+\left(1-v_{a}\left(k, a^{\mathrm{SB}}\right)\right) \epsilon_{v_{a a}, a}\left(k, a^{\mathrm{SB}}\right)}<0
$$

which, together with the application of the Envelope Theorem to Eq. (6), implies

$$
\begin{aligned}
& \frac{\partial^{2} S^{\mathrm{SB}}}{\partial k \partial \sigma^{2}}=-v_{a}\left(k, a^{\mathrm{SB}}\right) v_{a k}\left(k, a^{\mathrm{SB}}\right) \\
& {\left[r+\left(\frac{1}{v_{a}\left(k, a^{\mathrm{SB}}\right)}+\frac{r \sigma^{2}}{a^{\mathrm{SB}}}\left(\epsilon_{v_{a}, a}\left(k, a^{\mathrm{SB}}\right)+\epsilon_{v_{a k}, a}\left(k, a^{\mathrm{SB}}\right)\right)\right) \frac{\mathrm{d} a^{\mathrm{SB}}}{\mathrm{d} \sigma^{2}}\right]} \\
& =r v_{a}\left(k, a^{\mathrm{SB}}\right) v_{a k}\left(k, a^{\mathrm{SB}}\right)\left(-\epsilon_{a^{\mathrm{SB}}, \sigma^{2}}\left(k, a^{\mathrm{SB}}\right)\right) \epsilon \frac{v_{a k}, a}{v_{a a}}\left(k, a^{\mathrm{SB}}\right)
\end{aligned}
$$

where the last equality follows from Eq. (18) and Eq. (2) which implies $\frac{1}{v_{a}\left(k, a^{\mathrm{SB}}\right)} \frac{a^{\mathrm{SB}}}{r \sigma^{2}}=$ $\frac{v_{a}\left(k, a^{\mathrm{SB}}\right)}{1-v_{a}\left(k, a^{\mathrm{SB}}\right)} \epsilon_{v_{a}, a}\left(k, a^{\mathrm{SB}}\right)$. By Assumption $1, \frac{\partial^{2} S^{\mathrm{SB}}}{\partial \sigma^{2} \partial k} \geq(\leq) 0$ if and only if $\epsilon \frac{v_{a k}}{v_{a a}}, a\left(k, a^{\mathrm{SB}}\right)$ $\geq(\leq) 0$. Note that $a^{\mathrm{SB}}\left(k_{H}, \sigma_{H}^{2}\right)=\min \left\{a^{\mathrm{SB}}\left(k, \sigma^{2}\right) \mid\left(k, \sigma^{2}\right) \in K \times \Sigma\right\}$ and $\bar{a}^{\mathrm{SB}}\left(k_{L}, \sigma_{L}^{2}\right)=\max \left\{a^{\mathrm{SB}}\left(k, \sigma^{2}\right) \mid\left(k, \sigma^{2}\right) \in K \times \Sigma\right\}$ since $a^{\mathrm{SB}}$ is strictly decreasing in both $k$ and $\sigma^{2}$ by Eqs. (2) and (18). Let $\mu\left(\sigma^{2}\right)$ denote a measure-preserving matching function. Then, under NAM, $\min \left\{a^{\mathrm{SB}}\left(\mu\left(\sigma^{2}\right), \sigma^{2}\right) \mid \sigma^{2} \in \Sigma\right\} \geq a^{\mathrm{SB}}\left(k_{H}, \sigma_{H}^{2}\right)$ and $\max \left\{a^{\mathrm{SB}}\left(\mu\left(\sigma^{2}\right), \sigma^{2}\right) \mid \sigma^{2} \in \Sigma\right\} \leq a^{\mathrm{SB}}\left(k_{L}, \sigma_{L}^{2}\right)$. Therefore, the satisfaction of $\epsilon \frac{v_{a k}, a}{v_{a a}}\left(k, a^{\mathrm{SB}}\right) \leq 0$ for all $(k, a) \in K \times\left[a^{\mathrm{SB}}\left(k_{H}, \sigma_{H}^{2}\right), a^{\mathrm{SB}}\left(k_{L}, \sigma_{L}^{2}\right)\right]$ is a sufficient condition for NAM.

Proofs of Corollaries 6, 7 and 8 The proofs of Corollaries 6, 7 and 8 are analogous to the proofs of Corollaries 1, 2 and 3 but applying Proposition 6 instead of Proposition 1. The analogy is transparent so details are omitted.

Proof of Corollary 9 We examine the sign of Eq. (16). The sign of $\mathrm{d} a^{\mathrm{SB}} / \mathrm{d} \sigma^{2}$ is unambiguously negative by Eq. (18).

If the rate $v_{a k}(k, a) / v_{a a}(k, a)$ is weakly increasing in $a$ for all $(k, a) \in K \times$ $\left[a^{\mathrm{SB}}\left(k_{H}, \sigma_{H}^{2}\right), a^{\mathrm{SB}}\left(k_{L}, \sigma_{L}^{2}\right)\right]$ and strictly increasing in $a$ for some $(k, a) \in K \times$ $\left[a^{\mathrm{SB}}\left(k_{H}, \sigma_{H}^{2}\right), a^{\mathrm{SB}}\left(k_{L}, \sigma_{L}^{2}\right)\right]$, then: (i) we have a non-negative bias for all ability levels $\left(\lambda\left(k, a^{\mathrm{SB}}\right) \geq 0\right.$ for all $\left.k \in K\right)$, which implies that the second-best power $s^{\mathrm{SB}}$ is increasing in ability by Eq. (12) and Assumption 1; (ii) there is PAM in equilibrium by Proposition $6\left(\mu^{\prime}\left(\sigma^{2}\right) \geq 0\right)$. Thus, $-\left(v_{a k}\left(\mu\left(\sigma^{2}\right), a^{\mathrm{SB}}\right) / v_{a a}\left(\mu\left(\sigma^{2}\right), a^{\mathrm{SB}}\right)\right) \lambda\left(\mu\left(\sigma^{2}\right)\right.$, $\left.a^{\mathrm{SB}}\right) \mu^{\prime}\left(\sigma^{2}\right) \leq 0$ which implies $d s^{\mathrm{SB}} / d \sigma^{2}<0$ by Eq. (16) and Assumption 1.

Analogously, if the rate $v_{a k}(k, a) / v_{a a}(k, a)$ is weakly decreasing in $a$ for all $(k, a) \in$ $K \times\left[a^{\mathrm{SB}}\left(k_{H}, \sigma_{H}^{2}\right), a^{\mathrm{SB}}\left(k_{L}, \sigma_{L}^{2}\right)\right]$ and strictly decreasing in $a$ for some $(k, a) \in K \times$ $\left[a^{\mathrm{SB}}\left(k_{H}, \sigma_{H}^{2}\right), a^{\mathrm{SB}}\left(k_{L}, \sigma_{L}^{2}\right)\right]$ then: (i) we have a non-positive bias for all ability levels $\left(\lambda\left(k, a^{\mathrm{SB}}\right) \leq 0\right.$ for all $\left.k \in K\right)$ and the second-best power $s \mathrm{SB}$ is decreasing in ability by Proposition 1; (ii) there is NAM by Proposition $6\left(\mu^{\prime}\left(\sigma^{2}\right) \leq 0\right)$. Hence, $d s^{\mathrm{SB}} / d \sigma^{2}<0$ in Eq. (16) since $-\left(v_{a k}\left(\mu\left(\sigma^{2}\right), a^{\mathrm{SB}}\right) / v_{a a}\left(\mu\left(\sigma^{2}\right), a^{\mathrm{SB}}\right)\right) \lambda\left(\mu\left(\sigma^{2}\right), a^{\mathrm{SB}}\right) \mu^{\prime}\left(\sigma^{2}\right) \leq 0$ and $v_{a a}(a, k)>0$ by Assumption 1 . 
If the rate $v_{a k}(k, a) / v_{a a}(k, a)$ is invariant in $a$ for all $(k, a) \in K \times\left[a^{\mathrm{SB}}\left(k_{H}, \sigma_{H}^{2}\right)\right.$, $\left.a^{\mathrm{SB}}\left(k_{L}, \sigma_{L}^{2}\right)\right]$ then there is no bias for all ability levels $\left(\lambda\left(k, a^{\mathrm{SB}}\right)=0\right.$ for all $\left.k \in K\right)$, implying no indirect effect of risk on incentives. Thus, the total effect is equal to its negative direct effect given by Eq. (18).

\section{Appendix B: Supplementary appendix}

Corollary 10 Let us suppose that Assumption 1 holds. If the marginal COE function is homothetic and strictly quasiconcave for all $(k, a) \in K \times\left[a^{\mathrm{SB}}\left(k_{H}, r, \sigma^{2}\right), a^{\mathrm{FB}}\left(k_{L}\right)\right]$, then the efficiency loss increases with ability. If the marginal COE function is homothetic and strictly quasiconvex for all $(k, a) \in K \times\left[a^{\mathrm{SB}}\left(k_{H}, r, \sigma^{2}\right), a^{\mathrm{FB}}\left(k_{L}\right)\right]$, then the efficiency loss decreases with ability.

Proof From the proof of Corollary 1, if the marginal COE is homothetic and strictly quasiconcave for all $(k, a) \in K \times\left[a^{\mathrm{SB}}\left(k_{H}, r, \sigma^{2}\right), a^{\mathrm{FB}}\left(k_{L}\right)\right]$, then $\frac{v_{a k}(k, a)}{v_{a a}(k, a)}$ is strictly increasing in $a$ for all $(k, a) \in K \times\left[a^{\mathrm{SB}}\left(k_{H}, r, \sigma^{2}\right), a^{\mathrm{FB}}\left(k_{L}\right)\right]$. Thus, for any given $k \in K, \frac{v_{a k}(k, a)}{v_{a a}(k, a)}>\frac{v_{a k}\left(k, a^{\mathrm{SB}}\right)}{v_{a a}\left(k, a^{\mathrm{SB}}\right)}$ for all $a \in\left(a^{\mathrm{SB}}, a^{\mathrm{FB}}\right)$. The result is direct by applying Corollary 5. Analogously, if the marginal COE is homothetic and strictly quasiconvex for all $(k, a) \in K \times\left[a^{\mathrm{SB}}\left(k_{H}, r, \sigma^{2}\right), a^{\mathrm{FB}}\left(k_{L}\right)\right]$, then $\frac{v_{a k}(k, a)}{v_{a a}(k, a)}$ is strictly decreasing in $a$ for all $(k, a) \in K \times\left[a^{\mathrm{SB}}\left(k_{H}, r, \sigma^{2}\right), a^{\mathrm{FB}}\left(k_{L}\right)\right]$ by the proof of Corollary 1 . Hence, for any given $k \in K, \frac{v_{a k}(k, a)}{v_{a a}(k, a)}<\frac{v_{a k}\left(k, a^{\mathrm{SB}}\right)}{v_{a a}\left(k, a^{\mathrm{SB}}\right)}$ for all $a \in\left(a^{\mathrm{SB}}, a^{\mathrm{FB}}\right)$. By applying Corollary 5 , we get the result.

A less (more) restrictive condition is sufficient for a positive (negative) relationship between loss efficiency and ability under multiplicative separability of the marginal COE than under additive separability as the following Corollaries state.

Corollary 11 Let us suppose that Assumption 1 holds and suppose that the marginal COE function is multiplicatively separable. For any given ability level, if the marginal COE is log-concave (log-convex) in effort on $\left(a^{\mathrm{SB}}, a^{\mathrm{FB}}\right)$ then the efficiency loss increases (decreases) with ability.

Proof Assume that the marginal COE satisfies Assumption 1 and it is of the form $v_{a}(k, a)=g(k) f_{a}(a)$. Then,

$$
\begin{gathered}
\left(\frac{v_{a}\left(k, a^{\mathrm{FB}}\right)-v_{a}\left(k, a^{\mathrm{SB}}\right)}{v_{a a}\left(k, a^{\mathrm{SB}}\right)}\right)-\left(\frac{v_{k}\left(k, a^{\mathrm{FB}}\right)-v_{k}\left(k, a^{\mathrm{SB}}\right)}{v_{a k}\left(k, a^{\mathrm{SB}}\right)}\right) \\
=\left(\frac{f_{a}\left(a^{\mathrm{FB}}\right)-f_{a}\left(a^{\mathrm{SB}}\right)}{f_{a a}\left(a^{\mathrm{SB}}\right)}\right)-\left(\frac{f\left(a^{\mathrm{FB}}\right)-f\left(a^{\mathrm{SB}}\right)}{f_{a}\left(a^{\mathrm{SB}}\right)}\right)
\end{gathered}
$$

As a result, by Eq. (17), we have that:

$$
\frac{\partial L}{\partial k}=g^{\prime}(k) f_{a}\left(a^{\mathrm{SB}}\right) \int_{a^{\mathrm{SB}}}^{a^{\mathrm{FB}}}\left(\frac{f_{a a}(a)}{f_{a a}\left(a^{\mathrm{SB}}\right)}-\frac{f_{a}(a)}{f_{a}\left(a^{\mathrm{SB}}\right)}\right) \mathrm{d} a
$$


By definition, if the marginal COE is log-concave in $a$ for all $a \in\left(a^{\mathrm{SB}}, a^{\mathrm{FB}}\right)$, then $\frac{v_{a}(k, a)}{v_{a a}(k, a)}=\frac{f_{a}(a)}{f_{a a}(a)}$ is increasing in $a$ for $a \in\left(a^{\mathrm{SB}}, a^{\mathrm{FB}}\right)$. Thus, $\frac{f_{a}(a)}{f_{a a}(a)} \geq \frac{f_{a}\left(a^{\mathrm{SB}}\right)}{f_{a a}\left(a^{\mathrm{SB}}\right)}$ for all $a \in\left(a^{\mathrm{SB}}, a^{\mathrm{FB}}\right)$ implies $\frac{f_{a a}(a)}{f_{a a}\left(a^{\mathrm{SB}}\right)} \leq \frac{f_{a}(a)}{f_{a}\left(a^{\mathrm{SB}}\right)}$ for all $a \in\left(a^{\mathrm{SB}}, a^{\mathrm{FB}}\right)$, and hence, $\frac{\partial L}{\partial k} \leq 0$.

Analogously, if the marginal COE is log-convex in $a$ for all $a \in\left(a^{\mathrm{SB}}, a^{\mathrm{FB}}\right)$ then $\frac{v_{a}(k, a)}{v_{a a}(k, a)}=\frac{f_{a}(a)}{f_{a a}(a)}$ is decreasing in $a$ for $a \in\left(a^{\mathrm{SB}}, a^{\mathrm{FB}}\right)$. Thus, $\frac{f_{a}(a)}{f_{a a}(a)} \leq \frac{f_{a}\left(a^{\mathrm{SB}}\right)}{f_{a a}\left(a^{\mathrm{SB}}\right)}$ for all $a \in\left(a^{\mathrm{SB}}, a^{\mathrm{FB}}\right)$ implies $\frac{f_{a a}(a)}{f_{a a}\left(a^{\mathrm{SB}}\right)} \geq \frac{f_{a}(a)}{f_{a}\left(a^{\mathrm{SB}}\right)}$ for all $a \in\left(a^{\mathrm{SB}}, a^{\mathrm{FB}}\right)$, and hence, $\frac{\partial L}{\partial k} \geq 0$.

If the marginal COE function is additively separable then $v_{a k}(k, a) / v_{a a}(k, a)$ is of the form $g^{\prime}(k) / f_{a a}(a)$. Therefore, the convexity/concavity of the marginal COE on the interval $\left(a^{\mathrm{SB}}, a^{\mathrm{FB}}\right)$ determines the sign of the relationship by Corollaries 4 and 12 . Corollary 12 follows directly from Proposition 3 as well since the function $v_{k}(a, k)$ is linearly increasing in effort for all parameter values if the marginal COE function is additively separable.

Corollary 12 Let us suppose that Assumption 1 holds and suppose that the marginal COE function is additively separable. For any given ability level, if the marginal COE is convex in effort on $\left(a^{\mathrm{SB}}, a^{\mathrm{FB}}\right)$, then the efficiency loss decreases with ability, whereas if it is linear in effort on $\left(a^{\mathrm{SB}}, a^{\mathrm{FB}}\right)$, then the efficiency loss does not vary with ability.

Proof If the marginal COE is additively separable, then $v_{k}$ is linear in effort. The linearity of $v_{k}$ in effort implies $\frac{v_{k}\left(k, a^{\mathrm{FB}}\right)-v_{k}\left(k, a^{\mathrm{SB}}\right)}{v_{a k}\left(k, a^{\mathrm{SB}}\right)}=a^{\mathrm{FB}}-a^{\mathrm{SB}}$. If $v_{a}$ is also linear in effort for all $a \in\left(a^{\mathrm{SB}}, a^{\mathrm{FB}}\right)$, then $\frac{v_{a}\left(k, a^{\mathrm{FB}}\right)-v_{a}\left(k, a^{\mathrm{SB}}\right)}{v_{a a}\left(k, a^{\mathrm{SB}}\right)}=a^{\mathrm{FB}}-a^{\mathrm{SB}}=\frac{v_{k}\left(a^{\mathrm{FB}}, k\right)-v_{k}\left(a^{\mathrm{SB}}, k\right)}{v_{a k}\left(k, a^{\mathrm{SB}}\right)}$. By applying Eq. (17), we get that $\frac{\partial L}{\partial k}=0$. If $v_{a}$ is convex in effort for all $a \in\left(a^{\mathrm{SB}}, a^{\mathrm{FB}}\right)$, then $\frac{v_{a}\left(k, a^{\mathrm{FB}}\right)-v_{a}\left(k, a^{\mathrm{SB}}\right)}{v_{a a}\left(k, a^{\mathrm{SB}}\right)} \geq a^{\mathrm{FB}}-a^{\mathrm{SB}}=\frac{v_{k}\left(k, a^{\mathrm{FB}}\right)-v_{k}\left(k, a^{\mathrm{SB}}\right)}{v_{a k}\left(k, a^{\mathrm{SB}}\right)}$. By applying Eq. (17), we get that $\frac{\partial L}{\partial k} \geq 0$.

\section{References}

Ackerberg DA, Botticini M (2002) Endogenous matching and the empirical determinants of contract form. J Polit Econ 110(3):564-591. https://doi.org/10.1086/339712

Aggarwal RK, Samwick AA (1999) The other side of the trade-off: the impact of risk on executive compensation. J Polit Econ 107(1):65-105. https://doi.org/10.1086/250051

Bandiera O, Guiso L, Prat A, Sadun R (2015) Matching firms, managers, and incentives. J Labor Econ 33(3):623-681. https://doi.org/10.1086/679672

Chade H, Swinkels J (2020) The moral hazard problem with high stakes. J Econ Theory 187:105032. https:// doi.org/10.1016/j.jet.2020.105032

Chen P, Li S, Ye B (2018) Risk-sharing matching and moral hazard. Bull Econ Res 70(2):165-174. https:// doi.org/10.1111/boer.12139

Edmans A, Gabaix X (2011) The effect of risk on the CEO market. Rev Financ Stud 24(8):2822-2863. https://doi.org/10.1093/rfs/hhq153

Hilt E (2006) Incentives in corporations: evidence from the American whaling industry. J Law Econ 49(1):197-227. https://doi.org/10.1086/504057

Holmstrom B, Milgrom P (1987) Aggregation and linearity in the provision of intertemporal incentives. Econometrica 55(2):303-328. https://doi.org/10.2307/1913238

Kandilov I, Vukina T (2016) Salaries or piece rates: on the endogenous matching of harvest workers and crops. Econ Inquiry 54(1):76-99. https://doi.org/10.1111/ecin.12231 
Lazear EP, Oyer P (2012) Personnel economics. In: The handbook of organizational economics. Princeton University Press, pp 479-519. https://doi.org/10.2307/j.ctt1r2ggg.16

Legros P, Newman AF (2002) Monotone matching in perfect and imperfect worlds. Rev Econ Stud 69:925942. https://doi.org/10.1111/1467-937X.00231

Li F, Ueda M (2009) Why do reputable agents work for safer firms? Financ Res Lett 6(1):2-12. https://doi. org/10.1016/j.frl.2008.12.001

Macho-Stadler I, Pérez-Castrillo D (2018) Moral hazard: base models and two extensions: chap 16. In: Handbook of game theory and industrial organization, vol I. Edward Elgar Publishing, pp 453-485. https://doi.org/10.4337/9781785363283.00025

Macho-Stadler I, Pérez-Castrillo D (2020) Agency theory meets matching theory. SERIEs. https://doi.org/ $10.1007 / \mathrm{s} 13209-020-00215-3$

Prendergast C (2002) The tenuous tradeoff between risk and incentives. J Polit Econ 110(5):1071-1102. https://doi.org/10.1086/341874

Roth AE, Sotomayor MAO (1990) Two-sided matching: a study in game-theoretic modeling and analysis. Cambridge University Press. https://doi.org/10.1017/CCOL052139015X

Rothschild M, Stiglitz J (1976) Equilibrium in competitive insurance markets: an essay on the economics of imperfect information. Q J Econ 90(4):629-649. https://doi.org/10.2307/1885326

Serfes K (2005) Risk sharing vs. incentives: contract design under two-sided heterogeneity. Econ Lett 88(3):343-349. https://doi.org/10.1016/j.econlet.2005.02.020

Serfes K (2008) Endogenous matching in a market with heterogeneous principals and agents. Int J Game Theory 36:587-619. https://doi.org/10.1007/s00182-007-0109-y

Shapley LS, Shubik M (1971) The assignment game I: the core. Int J Game Theory 1(1):111-130. https:// doi.org/10.1007/BF01753437

Wulf J (2007) Authority, risk, and performance incentives: evidence from division manager positions inside firms. J Ind Econ 55(1):169-196. https://doi.org/10.1111/j.1467-6451.2007.00307.x

Publisher's Note Springer Nature remains neutral with regard to jurisdictional claims in published maps and institutional affiliations. 